\title{
Formation and Persistence of Oppositional Identities*
}

\author{
Alberto Bisin ${ }^{\dagger} \quad$ Eleonora Patacchini ${ }^{\ddagger} \quad$ Thierry Verdier ${ }^{\S} \quad$ Yves Zenou \\ March 27, 2009 (This version: April 21, 2011)
}

\begin{abstract}
We develop a dynamic model of identity formation that explains why ethnic minorities may choose to adopt oppositional identities (i.e. some individuals may reject or not the dominant culture) and why this behavior may persist over time. We first show that the prevalence of an oppositional culture in the minority group cannot always be sustained in equilibrium. Indeed, because the size of the majority group is larger, there is an "imposed" process of exposition to role models from the majority group that favors the diffusion of mainstream values in the minority community. In spite of this, an oppositional culture in the minority group can nevertheless be sustained in steady-state if there is enough cultural segmentation in terms of role models, or if the size of the minority group is large enough, or if the degree of oppositional identity it implies is high enough. We also demonstrate that the higher the level of harassment and the number of racist individuals in the society, the more likely an oppositional minority culture will emerge. We finally show that ethnic identity and socialization effort can be more intense in mixed rather than segregated neighborhoods.
\end{abstract}

Key words: Ethnicity, role models, peer effects, cultural transmission, racism.

JEL Classification: A14, J15

${ }^{*}$ Thanks to Andrew Clark for the Morissey's quote. We are grateful to the editor, Eran Yashiv, and two anonymous referees for helpful comments.

${ }^{\dagger}$ New York University, USA. E-mail: alberto.bisin@nyu.edu

‡Universita' di Roma "La Sapienza", Italy, and CEPR. E-mail: eleonora.patacchini@uniroma1.it

§Paris School of Economics (PSE), France, and CEPR. E-mail: verdier@pse.ens.fr

؟Stockholm University and Research Institute of Industrial Economics (IFN), Sweden, and CEPR. E-mail: yves.zenou@ne.su.se. 
Bengali, bengali / Bengali, bengali / No no no / He does not want to depress you/ Oh no no no no no / He only wants to impress you / Oh.. Bengali in platforms / He only wants to embrace your culture / And to be your friend forever. ['Bengali in Platform,' Morissey, Viva Hate, 1988, Reprise/Wea]

\section{Introduction}

In April 1992, when a mostly white jury acquitted four police officers accused in a videotaped of beating a black motorist, thousands of people in Los Angeles, mainly young black and Latino males, joined in what has often been characterized as a race riot. In the summer of 2001, ethnic riots occurred on the streets of towns and cities in the north of England (e.g., Oldham, Leeds, Burnley, Bradford), involving young British Asian men. More recently, in November 2005, riots emerged in Paris' suburbs, sparked by the accidental deaths of two Muslim teenagers, and then spread to 300 French towns and cities. Most of the rioters were the French-born children of immigrants from Arab and African countries.

These race and ethnic riots ${ }^{1}$ have all recently placed the issue of racial and ethnic identity at the forefront of political debate in the United States and in Europe. Identity is the result of an individual's choice, often the choice not to conform to the accepted norms but rather to different norms that characterize a social, ethnic, or religious group. ${ }^{2}$ Furthermore, ethnic identities can be "oppositional", that is, they require the rejection of the accepted norms of the majority group (Ainsworth-Darnell and Downey, 1998). This is the case, for instance, of the so-called "ghetto culture" in the United States (Wilson, 1987). Also, studies in the US have found that African American students in poor areas may be ambivalent about learning standard English and performing well at school because this may be regarded as "acting white" (Delpit, 1995; Fordham and Ogbu, 1986; Ogbu, 1997; Austen-Smith and Fryer, 2005; Selod and Zenou, 2006; Battu et al., 2007; Fryer and Torelli, 2010). ${ }^{3}$

\footnotetext{
${ }^{1}$ But also the terrorist attacks in the U.S. and Europe (September 11, the March 2004 Madrid train bombings, the July 2005 London bombings), the killing of the author of a documentary about Muslim immigrants by a young Dutch-Moroccan in Amsterdam on November 2004, the riots in many Muslim communities in February 2006 after the publications of vignettes representing the prophet Mohammed in a Danish newspaper, and several others.

${ }^{2}$ In this perspective, identity is related to conformity. Identity and conformity are nonetheless clearly distinct. Preferences for conformity in fact limit the choice of individuals, inducing them to conform e.g., to social norms of behavior (see e.g., Bernheim, 1994, and Akerlof, 1997).

${ }^{3}$ Such extreme preferences amongst ethnic group members may also stem from a lack of economic opportunity, discrimination or they may stem from a desire to display greater racial or religious solidarity (Akerlof and Kranton, 2000). Ihlanfeldt and Scafidi (2002) evoke a wish to share culture, prejudice against whites,
} 
Oppositional identities often produce significant economic and social conflicts, as in the case of the ethnic and race riots cited above. But how intense are oppositional identities? Which economic and sociological factors mostly contribute to their formation? In particular, does neighborhood segregation induce intense and oppositional identities? In this paper we attempt to provide some answers to these questions.

A large literature in economics, sociology and anthropology, documents how ethnic traits are transmitted from parents to children and how ethnic identity is adopted (see, in particular, Akerlof and Kranton, 2000; Alba, 1990; Bernal and Knight, 1993; Boyd and Richerson, 1985, Cavalli-Sforza and Feldman, 1981; Phinney, 1990). In our reading of the evidence, parents directly make various socialization choices, e.g., the rules and beliefs the family conforms to and how much time they spend with their children. Parents also realize that socialization is partially the product of the social interaction their children engage into, which they affect by choosing which neighborhood to live in, the school children attend, their social circle of friends and acquaintances, the civic/social clubs and churches they belong to, etc. The role of parents in the socialization of their own children is nonetheless limited by the children's pro-active role in choosing who to imitate and learn from, thereby directly shaping their own cultural identity. An individual's general identity, in the words of Akerlof and Kranton (2000, p. 720), "is bound to social categories; and individuals identify with people in some categories and differentiate themselves from those in others."

We model the formation of ethnic traits along these lines, that is, as a mechanism that interacts cultural transmission and socialization inside the family, ${ }^{4}$ peer effects and social interactions, and identity choice.

To be more precise, our model has three main components:

(i) Trait transmission from parent to child:

In period $t$, the child adopts his parent's identity with some probability, which a positive function of parental effort $\tau_{i}$. Parent also chooses the intensity of their own identity $\alpha_{i}$. The parent's choice of $\tau_{i}$ is determined altruistically, but with "imperfect empathy" in that the parent evaluates the child's future well-being as if the child has picked up the same trait

or expectations of unfavorable treatment by whites against non-whites in white neighborhoods. One could also think of the advantages that members of a minority group can derive from locating close to one another, thereby improving their access to 'ethnic goods' such as food, education or religious service, not to mention the ability to socially interact in their own language (Alesina and La Ferrara, 2005).

${ }^{4}$ See Bisin and Verdier $(2000,2001)$ for a formal study of cultural transmission, and Bisin, Topa, and Verdier (2004), Cohen Zada (2003), Jellal and Wolff (2002), and Patacchini and Zenou (2011a) for empirical studies of cultural transmission and socialization of, respectively, religious traits, altruism, and preferences for education. 
(oppositional or mainstream) as the parent himself.

(ii) Trait transmission from role model to child:

In the absence of successful parental transmission, the child can adopt the trait of a randomly picked role model in the society at large. In this perspective, $d$ represents the "segmentation" of society, which captures the populations from which a minority role model is chosen from. When $d=1$, the role model is drawn from society at large. When $d=0$ the role model is drawn only from the minority population.

(iii) Child's determination of the intensity of his trait, or his own identity:

Last, the intensity with which the child identifies himself with his trait is chosen by the child, along with a "good" or "bad" action. These two choices are referred to as an "identity choice." Choosing an identity of any kind is costly (denoted by $C\left(\alpha_{i}\right)$ ). But having a strong identity reduces psychological cost of interacting with others. The optimal choice of action is predetermined by assumption (mainstream individuals chooses the "good" action, oppositional ones chooses the "bad" action) but the optimal choice of intensity of identity will depend on $Q_{i}$, the probability of interacting with someone of a different type, and $I_{i}$, the psychological cost of this interaction. As a result, $Q_{i} \times I_{i}$ is the expected psychological cost of interacting with someone with a different value system.

We first show that the prevalence of an oppositional culture in the minority group (i.e. individuals who reject the mainstream values) can be sustained if and only if there is enough cultural segmentation in terms of role models, and/or the size of minority group is large enough, and/or the degree of oppositional identity it implies is high enough, and/or the socio-economic opportunity cost of the actions it prescribes is small enough. In this steadystate equilibrium, the socialization effort of oppositional parents is higher than that of the mainstream minority families. There is indeed an asymmetry between the two cultural traits, "mainstream" and "oppositional". Since the majority group individuals are by definition mainstreams, there is an "imposed" process of exposition to role models from that group. ${ }^{5}$ This tends, quite naturally, to favor the diffusion of the mainstream values into the minority community. Given that, in order to have a long run constant fraction of oppositional individuals, it has to be the case that their family socialization effort compensates for this asymmetric cultural bias. However, when the "imposed" socialization through the majority cultural model is strong enough, then there is no way for the oppositional culture to survive and there is, in that case, full assimilation of the minority group to the mainstream values.

We then show that it is possible (and we identify sufficient conditions on economic funda-

\footnotetext{
${ }^{5}$ This is referred to as the meeting bias in favor of the majority group (whites) in Currarini et al. (2009).
} 
mentals) that ethnic identity and socialization effort are more intense in mixed rather than in segregated neighborhoods. As a result, our analysis suggests that the effect of mixed neighborhood on identity formation and socialization might be quite complex and may generate in some cases perverse results. This is particularly so if mixed neighborhood are conducive of explicit acts of rejection on the part of the majority group.

The paper is organized as follows. In the next section, we discuss the contribution of our paper with respect to the literature. In Section 3, we present the main model. Section 4 deals with the cultural equilibrium analysis and some comparative statics results. Section 5 is devoted to the justification of our assumptions and modelling choices. In Section 6, we introduce the possibility of harassment of the minority group. Section 7 considers the joint cultural evolution of both racist or intolerant majority preferences and oppositional minority culture. Finally, Section 8 concludes, provides some empirical evidence and discusses some policy issues.

\section{Background and previous literature}

Our model is linked to several literatures that we would like to discuss now.

\subsection{Modeling ethnic identity}

A first feature of our model is the way we model ethnic identity. There are in fact different ways of modeling (ethnic) identity. Identity is a concept widely used in other disciplines but it is relatively new to economics. If we think of individual identity, then individuals will care about their own self-image. People have a desire to feel good about oneself, or hold onto a particular view of oneself (Bénabou and Tirole, 2006; Koszegi, 2006). If we think of social identity, then individuals care about their own self-image but their self-image depends on who they are within the society, which, in turn, depends on the established social norms (Akerlof and Kranton, 2000, 2010). ${ }^{6}$ This view is related to the social identity theory in social psychology (Tajfel, 1981; Turner, 1982). Feeling good about oneself depends on how you understand your social identity. To be more precise, Akerlof and Kranton (2000, 2010) incorporate the psychology and sociology of identity into an economic model of behavior. In the utility function they propose, identity is associated with different social categories and how people in these categories should behave. Their results suggest that people belong to

\footnotetext{
${ }^{6}$ Davis $(1995,2003,2004)$ and Sen $(1999,2000,2004)$ were among the first along with Akerlof and Kranton $(2000,2010)$ to introduce identity in economics.
} 
certain groups and wish to adopt the corresponding social identity by behaving according to the behavioral prescriptions of these groups. More recently, Horst et al. (2007), Kirman and Teschl $(2004,2006)$ have extended this notion of identity by proposing their own analysis of the economic agent's identity, which is motivated not by the self-interested choices, but by achieving consistency between one's characteristics and one's desired self-image through participation in different social groups.

To summarize, in the social identity literature, there are psychological costs from failing to conform to one's own group identity. If we think of ethnic identity, the "acting white" phenomenon mentioned in the introduction is a good example of this. This is similar to the conformity models (see, among others, Akerlof, 1980; Akerlof, 1997; Ballester et al., 2006; Battu et al., 2005; Bernheim, 1994; Glaeser and Scheinkman, 2001; Kandel and Lazear, 1992; Fershtman and Weiss, 1998; Patacchini and Zenou, 2011b) where it is failing to conform to own group identity that is costly. In some other models, (e.g. Shayo, 2009), the psychological cost comes from comparing the status of one's own group with that of another.

In our model, the psychological cost arises from interactions with others (different type), regardless of whether they are from the same group or not. For example, a very integrated (i.e. assimilated) ethnic minority will have a cost of interacting with a person from the same ethnic group if the latter has a much stronger ethnic identity. In the case of acting white, a very assimilated African American will have a cost of interacting with an African American having a strong "black" identity because they have different values. The former may like to study and have good grades and thus may suffer to interact with the latter who will accuse him of acting white and thus reject his behavior.

This view of identity is relatively known in the non-economic literature. These ideas have been expressed by the theories of multiculturalism (Glazer and Moynihan, 1970; Taylor and Lambert, 1996), and conflict (Bobo, 1999). According to this view, the group identity formation is a sort of cultural distinction mechanism that allows individuals to reduce the psychological costs associated with cultural differences. In economic terms, the concept of cultural distinction can be motivated in terms of negative social interactions across individuals belonging to different identified groups or types.

\subsection{Neighborhood effects}

A second feature of our model is that neighborhood matters since if the transmission of identity from parents fails, then the child picks up his "trait" from the neighborhood where he lives. Neighborhood models a la Bénabou (1993) and Durlauf (2004), for example, consider the dynamics of neighborhood formation as they interact with income determination via 
neighborhood effects on education. The logic of social interactions in a group appears very explicitly in models such as Durlauf (1996). This latter paper explores the dynamics of income inequality by studying the evolution of human capital investment and neighborhood choice for a population of families.

This class of models is conceptually similar to our analysis. We extend this logic in new directions, but our idea is not per se original. Indeed, our model makes different assumptions in that majority group members can have adverse effects on minority group members whereas the neighborhood models assume that rich neighbors always benefit poor ones. And the neighborhood models endogenize the neighborhood memberships. But the conceptual logic is quite similar.

\subsection{Dynamic models of identity formation}

A third feature of our model is the fact that identity formation is not static and depends on the dynamic of neighborhood composition where the child lives. There are, in fact, few models that analyze the dynamic of identity formation. Horst et al. (2007) and Bénabou and Tirole (2011) are notable exceptions.

Horst et al. (2007) explore the idea of an agent's personal identity of which his social identity is one aspect and in which the evolution of peoples' identities is stochastic. Their concept of identity is on a more personal level and suggests that people have desired selfimages of themselves that they wish to attain at some time in the future. Hence, individuals aim to transform their current individual characteristics into those of their self-image. They try to achieve this by joining social groups and adopting the typical characteristics of these groups. However, groups will be modified over time by the people joining them. This may induce individuals to revise their previous choices and eventually to move on and to choose different groups. The model thus presents an endogeneous interaction structure and offers an account of endogenous group formation as well as an endogenous evolution of personal identity.

Bénabou and Tirole (2011) develop a theory of moral behavior, individual and collective, based on a general model of identity in which people care about "who they are" and infer their own values from past choices. They can explain escalating commitments, in which someone who has built up enough of some economic or social asset -wealth, career, family, culture, etc.- continues to invest in it even when the marginal return no longer justifies it. Intuitively, a higher stock raises the stakes on viewing the asset as beneficial to one's long-run welfare, and the way to reassure oneself of its value is to keep investing. This leads to excessive specialization (e.g., work versus family) and persistence in unproductive tasks. 
Most strikingly, one can even be made worse off by a higher capital stock, as the escalatingcommitment mechanism leads to a treadmill effect in which increases in wealth, social status, or professional achievement induce a self-defeating pursuit of the belief that happiness lies in the accumulation of those same assets. Their model also sheds light on oppositional behaviors. When two identities are likely to compete later on for time or resources, investing in one depreciates the perceived value of the other. An agent with substantial capital vested in an insecure, hard-to-measure identity (e.g., cultural attachments) may therefore refrain from profitable investments in others (education, labor market integration), and even destroy valuable assets, ending up worse off.

Compared to these two approaches, the dynamic of identity formation in our model is quite different. Indeed, children receive an identity trait (in our model either "oppositional" or "mainstream") and then must decide how intense their identity within that trait is by trading off the costs of such an action with the benefits of it due to the reduction of the expected psychological cost of interacting with someone of a different value system. The dynamics of our model comes from the evolution over time of the fraction of the population with a certain trait ("oppositional" or "mainstream"), which depends on parental's effort in transmitting that trait and the identity intensity choice from the children.

\subsection{Theoretical models of oppositional identity}

Finally, our model is also related to the small theoretical literature on the formation of oppositional identities. Using their definition of ethnic identity (see above), Akerlof and Kranton (2010, Chap. 8) are able to explain why some blacks do reject the whites' norm and why some don't. In their framework, the losses in identity utility for a black to be an insider (i.e. to integrate and join the dominant majority) is the lack of acceptance by whites. This black person who decides to be an insider is denied self-respect because he does not fit the insider racial ideal (which is to be white). There are also losses in identity utility for a black to be an outsider (i.e. adopt an identity in opposition to the insiders). In that case, he loses self-respect not because of rejection by whites but because her outsider ideal tells him he should not be working for (or cooperating with) whites. Finally, there are externalities since a black worker who chooses to be an insider loses utility when other black workers chose to be outsiders (and vice versa). This model can explain why some blacks may reject the white ideal by, for example, dropping out of school at an early stage because it is rational to do so when the alternative is working in the white world and not being successful, which entails a great loss of utility.

Austen-Smith and Fryer (2005) propose an alternative explanation by focussing on the 
tension faced by individuals between signalling their type to the outside labor market and signalling their type to their peers: signals that induce high wages can be signals that induce peer rejection. Also Battu et al. (2007) show that some ethnic minorities may reject the white norm even if it implies a penalty in the labor market because they enjoy a high utility by being part of their group. Finally, De Martí and Zenou (2010) assume that two individuals from the same community always face a low linking cost of forming a relationship while the inter-community cost diminishes with the rate of exposure of each of them to the other community. Using the recent developments of network theory, they show that oppositional identity patterns can arise for a wide range of parameters, even when the inter-community costs are high.

Compared to this literature, we have three main contributions. First, all the models cited above are static and explain how and sometimes why an oppositional culture emerges. Here, we go further by explaining not only how and why but also the dynamics and persistence of oppositional identities. This is our first contribution and it is important for policy purposes because it can explain why, after three or four generations, North-African children in France are still not well-integrated and cause riots. Our second contribution consists in modelling parents' transmission of identity. Indeed, in our framework, not only peers' but also parents' investment are crucial to understand ethnic minority identity choices. Once more, this is important for policy purposes because it means that mixing people of different ethnicities can backfire since ethnic minority parents can overreact and invest more effort in transmitting their identity, which ultimately leads to less integration. Our third contribution is the modelling of the behavior of the majority group (i.e. racism and harassment), which can negatively react to oppositional identity behaviors. As it turns out, racism and integration have natural complementarities that may give rise to social multiplier effects and/or multiple social steady state equilibria. This has also important policy implications because it indicates that an optimal integration policy should also take into account the reaction of the majority group.

\section{The model}

Suppose that the population is of fixed size $N$ and composed of a majority group and a minority group. We denote by subscript $b$ the minority group, whose size is $N_{b}$, and by subscript $w$ the majority group, whose size is $N_{w}$ (with $N_{b}+N_{w}=N$ ), to which some individuals from the minority group might want to assimilate, i.e. share the same preferences and values. The two groups can be differentiated by some external attribute, such as the skin 
color, hair, size, etc., which is exogenous to the individual. On the other hand, preferences, values and identity are not exogenous and are affected by individuals' decisions. Let us denote by $q_{b}=N_{b} /\left(N_{b}+N_{w}\right)$ and $q_{w}=1-q_{b}$, the fraction of individuals from the minority and majority groups in the population. By definition $q_{b}<1 / 2$.

We consider the formation of cultural traits through a mechanism that interacts $(i)$ cultural transmission and socialization inside the family, (ii) social interactions and peer effects, via imitation and learning, and (iii) identity choice.

To begin with, we focus only on cultural transmission and identity choices of the minority group $b$ (with parents eventually spending effort to transmit their values and preferences). Within this minority group, there are two potential types of individuals: those who adopt the mainstream values and want to assimilate to the majority group, and those who reject the mainstream social norms. In other words, some individuals in the minority group may "choose" to adopt "oppositional" identities so that some individuals of that group may identify with the dominant culture and others may reject that culture. We refer to the first group as being "mainstream" (type $i=m$ ) and the other as being "oppositional" (type $i=o)$.

All individuals from the majority group are assumed to be mainstream while minorities can be either mainstream or oppositional. We model the formation of assimilation or "oppositional" culture as follows.

(i) Families are composed of one parent and a child (both without specified gender). All children are born without defined preferences or cultural traits, and are first exposed to their parent's trait. Cultural transmission inside the family to the parent's trait $i=m, o$, occurs with a probability that is the result of costly socialization effort on the part of the parent (see Bisin and Verdier, 2000, 2001).

(ii) If a child from a family with trait $i=m, o$ is not directly socialized, he interacts with peers, role models, and other cultural parents within his neighborhood where he is raised. As a consequence of such social interactions, the child adopts a cultural trait with a probability that depends on the cultural composition of his neighborhood.

(iii) After being socialized to a particular trait (directly or indirectly), the intensity with which an individual identifies to that trait (i.e. his cultural identity) is nonetheless his personal choice, that is, it is not transmitted by the family. Building on Akerlof and Kranton (2000), we emphasize here the idea that identity formation is strongly related to the identification to others on prescriptions on what "should" or "ought" to be done. From this, it follows that interacting with individuals that do not share these prescriptions and values generate psychological costs, as it creates some cognitive dissonance between oneself 
and the others. Choosing the intensity of an identity can then be conceptualized as a psychological defense to reduce these costs. By choosing a stronger identity, an individual tends to reaffirm the perception/feeling of his own preferences and values structure even when confronted with someone who does not share these values. Doing so is not immediate and may require psychological resources and efforts.

\subsection{Preferences}

Each individual has to decide between two possible actions $G$ ("good") and $B$ ("bad"). Action $G$ is what is "good" in terms of mainstream values while action $B$ is what is rejected by the majority group but can, of course, be "good" for the minority group. For example, one could have: $G=\{$ Wearing the Islamic veil $\}$ and $B=\{$ Not wearing the Islamic veil $\}$ or $G=\{$ Wearing western clothes $\}$ and $B=\{$ Wearing ethnic clothes $\}$.

We define preferences such that a mainstream minority individual always prefers $G$ to $B$ while the oppositional person always prefers $B$ to $G$. In particular, we assume that individual preferences of each type $(i=m, o)$ have a utility component defined on actions $\{G, B\}$ and characterized in the following way.

(i) A mainstream minority individual (type $m$ ) with intensity of identity $\alpha_{m} \in[0,1]$ has a utility component on actions $\{G, B\}$ given by:

$$
u_{m}\left(G, \alpha_{m}\right)=U \text { and } u_{m}\left(B, \alpha_{m}\right)=-\Psi\left(\alpha_{m}\right)
$$

where $U>0$ reflects the economic returns of action $G$ while action $B$ generates a lower return, normalized to 0 for simplicity. There is also a cost $\Psi\left(\alpha_{m}\right)>0$ of choosing action $B$, which depends on $\alpha_{m} \in[0,1]$, a measure of the intensity of identity. It is assumed that $\Psi^{\prime}\left(\alpha_{m}\right)>0$ and $\Psi^{\prime \prime}\left(\alpha_{m}\right) \geq 0$, namely the stronger the identity associated to this "socially established" preference, the larger, and at an increasing rate, is the perceived "disutility" of action $B$. Since $u_{m}\left(G, \alpha_{m}\right)>u_{m}\left(B, \alpha_{m}\right), \forall \alpha_{m} \in[0,1]$, action $G$ is always chosen by a mainstream minority individual.

(ii) An "oppositional" minority individual with identity intensity $\alpha_{o}$, has an instantaneous utility on actions $\{G, B\}$ given by:

$$
u_{o}\left(G, \alpha_{o}\right)=U-\Phi\left(\alpha_{o}\right) \text { and } u_{o}\left(B, \alpha_{o}\right)=0
$$

where $\Phi\left(\alpha_{o}\right)$ is a disutility cost of action $G$ in that system of values and $\alpha_{o} \in[0,1]$ is the intensity of identity associated with that system of "oppositional" values. Again we assume that $\Phi^{\prime}\left(\alpha_{o}\right)>0, \Phi^{\prime \prime}\left(\alpha_{o}\right) \geq 0$, namely the stronger the identity associated to the 
"oppositional" preference, the larger, and at an increasing rate, the perceived "disutility" for undertaking action $G$. We will also make the following (simplifying) assumption that $U<\Phi(0)$ so that $u_{o}(G)<u_{o}(B), \forall \alpha_{o} \in[0,1]$, and thus action $B$ is always chosen by an oppositional minority. Here, individuals are "oppositional" in the sense that they value more action $B$, which is the action "not promoted" by the majority group value system.

Now, each individual $i=m, o$ not only decides which action $x=G, B$ to take, but also the intensity of his identity $\alpha_{i}$. More precisely, an individual of type $i=m$, o has the following instantaneous preferences:

$$
W_{i}\left(x, \alpha_{i}\right)=u_{i}\left(x, \alpha_{i}\right)-\lambda\left(\alpha_{i}\right) Q_{i} I_{i}-C\left(\alpha_{i}\right) \text { for } x=G, B
$$

where $u_{i}\left(x, \alpha_{i}\right)$ is the utility component defined on actions $x=G, B$, as described by (1) for $i=m$ and by (2) for $i=o$. In (3), there are two additional components. The term $\lambda\left(\alpha_{i}\right) Q_{i} I_{i}$ reflects the "social" utility loss for individual $i$ of interacting with individuals $j \neq i$. In this expression, $I_{i}$ and $Q_{i}$, are respectively the psychological cost and the probability for an individual of type $i=m$, of interacting with an individual of type $j=m, o, j \neq i^{7}$ while $\lambda\left(\alpha_{i}\right)$ is the unit cost for individual $i$ of not identifying to the value system $j \neq i$. Hence $\lambda\left(\alpha_{i}\right) Q_{i} I_{i}$ represents the expected psychological costs for individual $i$ of interacting with individual $j \neq i$. As stated in the Introduction, this is quite different from the conformist model since the psychological cost arises from interactions with others of a different type, regardless of whether they are from the same ethnic group or not. For example, a very assimilated African American in the United States will have a psychologic cost of interacting with an African American having a strong "black" identity because they have different values. The former may like to study and have good grades and thus may suffer to interact with the latter, who may accuse him of "acting white" and reject his behavior.

The unit cost $\lambda\left(\alpha_{i}\right)$ is, quite naturally, assumed to be decreasing with the intensity of identity, i.e. $\lambda^{\prime}\left(\alpha_{i}\right)<0$ (we also assume $\lambda^{\prime \prime}\left(\alpha_{i}\right) \geq 0$ to get a well defined concave problem, and $\lambda(0)=+\infty$ to get interior solutions for identity intensity). Let us give the intuition of why $\lambda^{\prime}\left(\alpha_{i}\right)<0$. The idea is that each minority individual $i=m, o$ has a psychological cost of interacting with another minority individual $j=m, o, j \neq i$, with whom he is randomly matched. However, a stronger identification with his own culture (i.e. higher $\alpha_{i}$ ) is a way of reducing this cost. Indeed, the more an individual is, for example, "oppositional", the more he is "pride" of his own ethnic identity, and the less he feels threatened by another minority individual who promotes mainstream (white) values.

Finally in (3), the term $C\left(\alpha_{i}\right)$ captures the fact that identity formation is costly in

\footnotetext{
${ }^{7} Q_{i}$ is determined below.
} 
itself. Hence higher values of $\alpha_{i}$ are formed at a psychological $\operatorname{cost} C\left(\alpha_{i}\right)$, with $C^{\prime}\left(\alpha_{i}\right)>0$, $C^{\prime \prime}\left(\alpha_{i}\right) \geq 0, C(0)=0$.

It is important here to differentiate how $\alpha_{i}$ affects the utility function $u_{i}\left(x, \alpha_{i}\right)$ and how it affects the psychological cost $\lambda\left(\alpha_{i}\right)$. Take again an oppositional individual $i=o$. For the former, a high $\alpha_{o}$ penalizes more action $G$ that is promoted by the mainstream culture. For the latter, a high $\alpha_{o}$ reduces the cost of interacting with a mainstream individual.

\subsection{Socialization and identity choice}

In a given period $t$, consider one of our minority group families composed of a parent of trait $i$ and a child. At the beginning of that period $t$, we assume that the adult parent $i$ has already been socialized to a value system $i=m, o$. He chooses his own identity $\alpha_{i}$ as well as his best action $x$. Then, he gives birth to his child who has not yet a well-defined value system. The adult parent chooses at this stage $\tau_{i}$, the direct socialization of his child. The child then, in turn, possibly acquires his particular trait $i$ through an intergenerational transmission mechanism, which depends on the parent's socialization effort, $\tau_{i}$, and on the social environment where the parent lives and the child is raised. Then, comes the next period $t+1$ with the child grown up as a socialized adult who faces the same sequence of events and actions as his parent in period $t$.

\subsubsection{The parent's and the child's identity choices}

As already mentioned above, in each period, an adult individual of type $i=m, o$ makes an identity choice, which consists in choosing action $x=G, B$ and identity intensity $\alpha_{i}$. We formulated preferences in such a way that mainstream minority individual (type $m$ ) always choose action $G$ while oppositional individuals (type $o$ ) always choose action $B$ (see (1) and (2)). So we have now to determine the choice of $\alpha_{i}$. A mainstream individual solves the following program

$$
\max _{\alpha_{m}}\left[U-\lambda\left(\alpha_{m}\right) Q_{m} I_{m}-C\left(\alpha_{m}\right)\right]
$$

while, for an oppositional individual, it is given by:

$$
\max _{\alpha_{o}}\left[-\lambda\left(\alpha_{o}\right) Q_{o} I_{o}-C\left(\alpha_{o}\right)\right]
$$

Solving both programs leads to the following first order conditions:

$$
-\lambda^{\prime}\left(\alpha_{i}^{*}\right) Q_{i} I_{i}-C^{\prime}\left(\alpha_{i}^{*}\right)=0, \quad i=m, o
$$


Observe that $-\lambda^{\prime}\left(\alpha_{i}\right)>0$ and $-C^{\prime}\left(\alpha_{i}\right)<0$, and since $\lambda^{\prime \prime}() \geq 0,. C^{\prime \prime}() \geq$.0 , and $\lambda(0)=$ $+\infty$, there is a unique interior solution to each of these problems. We denote this solution by $\alpha_{i}^{*}=\alpha\left(Q_{i} I_{i}\right)$, which depends positively on the expected identity cost $Q_{i} I_{i}$ of socially interacting with someone having a different value system.

\subsubsection{The parent's socialization choice}

The cultural transmission and socialization process we adopt here is similar to that of Bisin and Verdier $(2000,2001)$. Parents are altruistic but in a paternalistic manner. That is, parents care about their child's future well-being but they evaluate it as if it were their own. Bisin and Verdier $(2000,2001)$ refer to this form of paternalistic altruism in the context of cultural transmission models as imperfect empathy. So, for example, when a parent $i=m, o$ wants to evaluate the (future) utility of his child, he considers the following utility:

$$
V_{i}\left(x_{c}, \alpha_{i}\right)=u_{i}\left(x_{c}, \alpha_{i}\right)-\lambda\left(\alpha_{i}\right) Q_{c}^{e} I_{i}
$$

where $x_{c}$ represents the child's decisions ${ }^{8}$ of choosing between actions $G$ or $B$, given the child's own environment, and $Q_{c}^{e}$ denotes the probability of interactions between two individuals with different value systems in the child's environment, as expected by the parent. ${ }^{9}$ Therefore, while parents perceive and evaluate altruistically the behavior of their children, $x_{c}$, through the lenses of their own identity $\alpha_{i}$, they anticipate correctly the environment of their child (possibly different from their own environment), his choice $x_{c}$ and his probability of interactions $Q_{c}^{e}$ with individuals different from him. ${ }^{10}$ Clearly, the child optimal choice of action may be different from that of the parent. As such, this will be perceived by the parent as sub-optimal, given that the latter only evaluates his child's action through the lenses of his own perspective. This, in turn, will generate an incentive for the parent to socialize his child to his own value system.

To be more precise, consider a parent of trait $i=m, o$. He first decides how much effort $\tau_{i}$ he puts in direct vertical socialization. As a consequence, the child is directly socialized to trait $i$ with probability $\tau_{i}$. If the child is not directly socialized (which happens with probability $1-\tau_{i}$ ), he picks a cultural parent at random from the population of role models

\footnotetext{
${ }^{8}$ Subscript $c$ refers to the child. When a variable has no subscript $c$, then it corresponds to the choice variable of the parent.

${ }^{9}$ The superscript $e$ refers to the parent's expectations.

${ }^{10}$ Note also that parents do not take into account the cost $C\left(\alpha_{i}^{c}\right)$ of identity formation of the child. This is perfectly consistent with the fact that the parent perceives the child's actions through the lense of his own already formed identity intensity $\alpha_{i}$.
} 
in the neighborhood in which he is raised. The child thus adopts the trait $i=m, o$ if the role model happens to have this trait. Otherwise the child assimilates to the other value system. Therefore, the probability that a parent of trait $i$ has a child of trait $i$ is given by:

$$
P_{i i}=\tau_{i}+\left(1-\tau_{i}\right) r_{i}
$$

where $r_{i}$ is the probability that the society role model is of type $i{ }^{11}$ On the other hand, when not successfully socialized to trait $i$, the probability that the child does adopt the alternative trait $j \neq i$ is equal to:

$$
P_{i j}=\left(1-\tau_{i}\right)\left(1-r_{i}\right)
$$

Note that $P_{i i}$ and $P_{i j}$ both depend on $r_{i}$, the composition of role models in the neighborhood where the child is brought up. The parent $i$ 's socialization problem consists in solving the following program:

$$
\max _{\tau_{i}}\left\{P_{i i}\left[u_{i}\left(x_{i c}, \alpha_{i}^{*}\right)-\lambda\left(\alpha_{i}^{*}\right) Q_{c}^{e} I_{i}\right]+P_{i j}\left[u_{i}\left(x_{j c}, \alpha_{i}^{*}\right)-\lambda\left(\alpha_{i}^{*}\right) Q_{c}^{e} I_{i}\right]-\Theta\left(\tau_{i}\right)\right\}
$$

where $P_{i i}$ and $P_{i j}$ are given by $(7)$ and (8), $\alpha_{i}^{*}=\alpha\left(Q_{i} I_{i}\right)$ by $(6)$, and $\Theta\left(\tau_{i}\right)$ is the cost of socialization with $\Theta^{\prime}\left(\tau_{i}\right)>0$ for all $\tau_{i} \in(0,1], \Theta^{\prime \prime}\left(\tau_{i}\right) \geq 0, \Theta(0)=0$. To better understand this maximization problem, let us write it for each type of parent $i=m, o$. We have:

$$
\begin{gathered}
\max _{\tau_{m}}\left\{P_{m m}\left[U-\lambda\left(\alpha_{m}^{*}\right) Q_{c}^{e} I_{m}\right]+P_{m o}\left[-\Psi\left(\alpha_{m}^{*}\right)-\lambda\left(\alpha_{m}^{*}\right) Q_{c}^{e} I_{m}\right]-\Theta\left(\tau_{m}\right)\right\} \\
\max _{\tau_{o}}\left\{P_{o o}\left[-\lambda\left(\alpha_{o}^{*}\right) Q_{c}^{e} I_{o}\right]+P_{o m}\left[U-\Phi\left(\alpha_{o}^{*}\right)-\lambda\left(\alpha_{c}^{*}\right) Q_{c}^{e} I_{o}\right]-\Theta\left(\tau_{o}\right)\right\}
\end{gathered}
$$

Indeed, each parent evaluates the utility of his child as if it was his own utility (imperfect empathy). When the parent is of type $m$ (mainstream; see (10)), with probability $P_{m m}$ his child adopts the same trait $m$ and thus becomes mainstream. In that case, the child will always choose action $G$ (and thus gets utility $u_{m}(G)=U$ ) while he will be perceived as getting a utility loss of social interactions $\lambda\left(\alpha_{m}^{*}\right) Q_{c}^{e} I_{m}$ as evaluated by the parent, with identity of the child as $\alpha_{m}^{*}=\alpha\left(Q_{m} I_{m}\right)$ defined by (6). When making his socialization decision $\tau_{m}$, the parent anticipates that the percentage of individuals with trait $m$ living in the neighborhood of his child is $Q_{c}^{e}$. However, with probability $P_{m o}$, his child adopts the other trait $i=o$ and becomes oppositional. In that case, the parent anticipates that his child will always choose action $B$. This is where the imperfect empathy assumption comes in. If the parent was just evaluating the utility of his child, we would have had $u_{o}(B)=0$. But since he is evaluating the utility of his child through his own utility, we have: $u_{m}(B)=-\Psi\left(\alpha_{m}^{*}\right)$,

\footnotetext{
${ }^{11} r_{i}$ is determined below.
} 
i.e. taking action $B$ (the child) given that the type is $m$ (the parent). The interpretation of the other equation (11) is exactly the same with $u_{o}(B)=0$ for $P_{o o}$ and $u_{o}(G)=U-\Phi\left(\alpha_{o}^{*}\right)$ for $P_{o m}$.

The first order conditions of these problems yield:

$$
\Theta^{\prime}\left(\tau_{i}^{*}\right)=\left(1-r_{i}\right) \Delta V_{i}
$$

where for $i \neq j$,

$$
\Delta V_{i}=u_{i}\left(x_{i c}, \alpha_{i}^{*}\right)-u_{i}\left(x_{j c}, \alpha_{i}^{*}\right)
$$

and

$$
\begin{gathered}
\Delta V_{m}=U+\Psi\left(\alpha_{m}^{*}\right)=U+\Psi\left(\alpha\left(Q_{m} I_{m}\right)\right)>0 \\
\Delta V_{o}=\Phi\left(\alpha_{o}^{*}\right)-U=\Phi\left(\alpha\left(Q_{o} I_{o}\right)\right)-U>0
\end{gathered}
$$

$\Delta V_{i}$ captures parent $i$ 's identity since it measures how important it is for him that his child adopts his own trait $i$. For example, $\Delta V_{o}$ corresponds to parent $i$ 's perceived utility gains from having a non-assimilated child. Let $\tau_{i}^{*}=\tau_{i}\left(\Delta V_{i}\right)$ denote the parent's socialization choice, the solution of socialization problem $(9)$.

To close the model, we need to determine $Q_{i}$, the probability of interacting (in the parents' generation period) with somebody with the other value system, and $r_{i}$, the frequency of role models of type $i$ to whom a child of the minority group is exposed. Let $p_{b}$ (resp. $p_{w}$ ) be the probability for an individual from the minority group of being matched with someone from the minority group (resp. the majority group). It is respectively given by:

$$
\begin{aligned}
p_{b} & =q_{b}+(1-s) q_{w} \\
& =1-s\left(1-q_{b}\right)
\end{aligned}
$$

and

$$
p_{w}=s q_{w}
$$

where $q_{b}=N_{b} /\left(N_{b}+N_{w}\right)$ and $q_{w}=1-q_{b}$ denote respectively the fraction of minority and majority individuals in the population, while $s \in[0,1]$ is an inverse measure of the degree of social segmentation or segregation between the minority and the majority groups. When $s=0$, there is full segmentation/segregation and thus $p_{b}=q_{b}+q_{w}$ and $p_{w}=0$. The case $s=1$ corresponds to a random matching since $p_{b}=q_{b}$ and $p_{w}=q_{w}$. Therefore, the lower is $s$, the more the society is segregated and it is less likely for the different communities to interact with each other $\left(\frac{\partial p_{b}}{\partial s}<0\right.$ and $\left.\frac{\partial p_{w}}{\partial s}>0\right)$. Assuming that, within the minority 
group, matching is random, we immediately obtain the probabilities $Q_{i}$ of interacting with individuals $j \neq i$ as:

$$
\begin{gathered}
Q_{m}=p_{b}\left(1-q_{m}\right)=\left[q_{b}+(1-s) q_{w}\right]\left(1-q_{m}\right) \\
Q_{o}=p_{b} q_{m}+p_{w}=\left[q_{b}+(1-s) q_{w}\right] q_{m}+s q_{w}
\end{gathered}
$$

where $q_{m}=N_{m} / N_{b}$ and $1-q_{m}$ are respectively the fractions of mainstream and oppositional individuals in the minority group, and where $N_{m}$ is the number of mainstream minority individuals. Since a person from the majority group cannot be oppositional, the probability $Q_{m}$ that a mainstream minority individual meets an oppositional individual is simply $p_{b}\left(1-q_{m}\right)$, i.e. the probability of meeting an oppositional individual within the minority community. However, $Q_{o}$, the probability that an oppositional individual meets a mainstream individual is the sum of two probabilities, namely $p_{b} q_{m}$, the probability to be matched with a mainstream individual from the minority community plus $p_{w}$ the probability of being matched with a majority individual (who by definition is a mainstream person). Since $q_{b}+q_{w}=1$, we have:

$$
\begin{gathered}
Q_{m}=\left(1-s+s q_{b}\right)\left(1-q_{m}\right) \\
Q_{o}=\left(1-s+s q_{b}\right) q_{m}+s\left(1-q_{b}\right)
\end{gathered}
$$

with

$$
\begin{aligned}
Q_{m} & =Q_{m}\left(\bar{q}_{m}^{-}, \bar{s}, \stackrel{+}{q_{b}}\right) \\
Q_{o} & =Q_{o}\left({\stackrel{+}{q_{m}}}_{m}, \stackrel{+}{s}, \overline{q_{b}}\right)
\end{aligned}
$$

and

$$
Q_{m}\left(q_{m}, s, q_{b}\right)+Q_{o}\left(q_{m}, s, q_{b}\right)=1
$$

We now need to determine $r_{i}$, the probability for a child to being exposed to a role model of type $i$. The frequency of role models of type $m$ to which a child from the minority group is exposed to depends on the technology of information, the socialization of the society, and the community at large (schools, churches, clubs, gangs, etc...). If we assume that there is also a degree of segmentation $d \in[0,1]$ in cultural exposure along the minority-majority lines (possibly different from $s$ ), one may then write the following probabilities as:

$$
\begin{gathered}
r_{m}=\left[q_{b}+(1-d) q_{w}\right] q_{m}+d q_{w}=\left(1-d+d q_{b}\right) q_{m}+d\left(1-q_{b}\right) \\
r_{o}=\left[q_{b}+(1-d) q_{w}\right]\left(1-q_{m}\right)=\left(1-d+d q_{b}\right)\left(1-q_{m}\right)
\end{gathered}
$$


Indeed, the probability $r_{m}$ for a child of meeting a mainstream role model is, once more, the sum of two probabilities, namely the probabilities of meeting a mainstream role model among the minority and majority groups. For $r_{o}$, since only ethnic minorities can be oppositional, it is simply the probability of meeting an oppositional role model among the minority group. Observe that when $d=0$, there is full segmentation since $r_{m}=q_{m}$ and $r_{o}=1-q_{m}$ whereas the case $d=1$ corresponds to a random matching since $r_{m}=1-r_{o}$ and $r_{o}=q_{b}\left(1-q_{m}\right)$. Therefore, the higher is $d$, the less the society is culturally segmented between the majority and the minority groups and it is more likely for ethnic minorities to meet mainstream role models $\left(\frac{\partial r_{m}}{\partial d}>0\right.$ and $\left.\frac{\partial r_{o}}{\partial d}<0\right)$. Simple inspection immediately gives:

$$
\begin{aligned}
& r_{m}=r_{m}\left(\stackrel{+}{q}_{m}, \stackrel{+}{d}, \bar{q}_{b}\right) \\
& r_{o}=r_{o}\left(\bar{q}_{m}, \bar{d}, \stackrel{+}{q}_{b}\right)
\end{aligned}
$$

Namely, $r_{o}$, the probability to be exposed to an oppositional role model is an increasing function of the degree of segmentation (low value of $d$ ), the fraction of the minority group (high value of $q_{b}$ ), and the fraction of oppositional individuals in the minority group (high value of $\left.1-q_{m}\right)$. For $r_{m}=1-r_{o}$, we have, of course, the opposite result.

We are now able to write the socialization effort decision $\tau_{i}$ of parents as a function of $q_{m}, q_{b}, s, d, U$. Indeed, (12) can now be written as:

$$
\Theta^{\prime}\left(\tau_{i}^{*}\right)=\left[1-r_{i}\left(q_{m}, d, q_{b}\right)\right] \Delta V_{i}\left(U, Q_{i}\left(q_{m}, s, q_{b}\right) I_{i}\right)
$$

Using (18), (19), (22), and (23), we easily obtain:

$$
\begin{gathered}
\tau_{m}^{*}=\tau_{m}\left(\bar{q}_{m}, \stackrel{+}{q_{b}}, \bar{s}, \bar{d}, \stackrel{+}{U}\right) \\
\tau_{o}^{*}=\tau_{o}\left(\stackrel{+}{q}_{m}, \bar{q}_{b}, \stackrel{+}{s}, \stackrel{+}{d}, \bar{U}\right)
\end{gathered}
$$

The cultural transmission effort $\tau_{m}$ of the mainstream minority family is a decreasing function of the fraction of mainstream individuals, whether inside the minority group (fraction $q_{m}$ ) or the majority group (fraction $q_{w}=1-q_{b}$ ). We have the opposite result for oppositional parents. This reflects cultural substituability between family and external role models in socializing children to a particular cultural trait. Indeed, the more common is a trait $i=m, o$ in the society, the lower is the parents' effort in socializing that trait. Moreover, the cultural transmission effort $\tau_{m}$ of mainstream minority parents is increasing with segregation (low values of $s$ ). Indeed, when segregation between the majority and the minority groups is very 
severe, i.e. the two groups do not interact very much with each other, the mainstream minority parent tends to mostly interact with oppositional minority parents and has therefore higher incentives to develop a strong mainstream identity $\alpha_{m}$. This, in turn, increases his marginal incentives to have his child adopting his own preference pattern (i.e. $\Delta V^{m}$ ) and therefore his socialization effort $\tau_{m}$ is larger. Finally the larger the degree of cultural segmentation (low values of $d$ ), i.e. the less likely for ethnic minorities to meet mainstream role models, the larger the socialization effort of the mainstream minority individual. Again, this reflects the cultural substituability effect. High cultural segmentation means that children of mainstream minority families have more chance to be exposed to oppositional role models. This, in turn, induces mainstream minority families to spend more effort to "preserve" their children from such cultural influence. Similar intuition can be developed to understand the effects of $q_{m}, q_{b}, s$, and $d$ on the cultural transmission effort $\tau_{o}^{*}$ of oppositional families.

\section{Cultural equilibrium and its properties}

\subsection{Steady-state equilibrium}

The dynamics of cultural evolution from period $t$ to period $t+1$ is described by the following equation :

$$
\begin{aligned}
q_{m, t+1} & =q_{m, t} P_{m m}+\left(1-q_{m, t}\right) P_{o m} \\
& =q_{m, t}\left[\tau_{m t}^{*}+\left(1-\tau_{m t}^{*}\right) r_{m t}\right]+\left(1-q_{m, t}\right)\left(1-\tau_{o t}^{*}\right) r_{m t}
\end{aligned}
$$

where $\tau_{i t}^{*}=\tau_{i}\left(q_{m, t}\right)$ is defined by $(24)$ and $r_{m t}=r_{m}\left(q_{m, t}\right)$ by $(20)$. The interpretation of this equation is straightforward. The proportion of mainstream minority individuals $q_{m, t+1}$ at time $t+1$ is equal to all new-born minority children who become mainstream and whose parents were mainstream $\left(q_{m, t} P_{m m}\right)$ plus all new-born minority children who become mainstream but whose parents were oppositional $\left(\left(1-q_{m, t}\right) P_{o m}\right)$.

This equation is equivalent to:

$$
q_{m, t+1}-q_{m, t}=\left(1-q_{m, t}\right)\left(1-\tau_{o t}^{*}\right) r_{m t}-\left(1-\tau_{m t}^{*}\right) q_{m, t}\left(1-r_{m t}\right)
$$

Now, by substituting the values of $r_{m}$ and $1-r_{m}$ from (20) in this equation, we easily obtain:

$$
q_{m, t+1}-q_{m, t}=\left(1-q_{m, t}\right)\left\{q_{m, t}\left(1-d+d q_{b}\right)\left(\tau_{m t}^{*}-\tau_{o t}^{*}\right)+\left(1-\tau_{o t}^{*}\right) d\left(1-q_{b}\right)\right\}
$$

In steady-state, $q_{m, t+1}=q_{m, t}=q_{m}^{*}$, and we have: 


$$
\left(1-q_{m}^{*}\right)\left\{q_{m}^{*}\left(1-d+d q_{b}\right)\left(\tau_{m}^{*}-\tau_{o}^{*}\right)+\left(1-\tau_{o}^{*}\right) d\left(1-q_{b}\right)\right\}=0
$$

There is a first obvious steady-state equilibrium at $q_{m}^{*}=1$, which implies that all minority individuals fully assimilate to the mainstream values. There can also be an interior steadystate equilibrium defined as follows:

$$
\Omega\left(q_{m}^{*}\right)=q_{m}^{*}\left(1-d+d q_{b}\right)\left(\tau_{m}^{*}-\tau_{o}^{*}\right)+\left(1-\tau_{o}^{*}\right) d\left(1-q_{b}\right)=0
$$

The following proposition characterizes the nature of the long-run cultural steady-state equilibrium: ${ }^{12}$

Proposition 1 (Existence and uniqueness of an interior equilibrium) There exists a unique interior steady-state equilibrium $\left.q_{m}^{*} \in\right] 0,1[$ defined by (27) if and only if:

$$
\Theta^{\prime}\left(d\left(1-q_{b}\right)\right)<\Phi\left(\alpha_{o}\left(I_{o}\right)\right)-U
$$

In such a steady-state, the oppositional minority families exert more cultural transmission effort than the mainstream minority families, i.e. $\tau_{m}^{*} \leq \tau_{o}^{*}$.

Proposition 1 essentially says that an oppositional culture in the minority group can be sustained if and only if there is enough cultural segmentation in terms of role models (i.e. $d$ small enough), and/or the size of minority group is large enough (i.e. $q_{b}$ large enough), and/or the degree of oppositional identity it implies is high enough (i.e. $\Phi($.$) and I_{o}$ high enough), and/or the socio-economic opportunity cost of the actions it prescribes is small enough (i.e. $U$ small enough). Note that there is clearly an asymmetry between the two cultural traits $m$ and $o$ because of the "imposed" process of exposition to role models from the dominant majority group. This tends quite naturally to favor the diffusion of the mainstream values into the minority community. Given that, in order to have a long run constant fraction of oppositional individuals, it has to be that their family socialization effort compensates for this asymmetric cultural bias. This is why their socialization effort $\tau_{o}^{*}$ is larger than that of the mainstream minority families, $\tau_{m}^{*}$. When the "imposed" socialization through the majority cultural model is strong enough, then there is no way for the oppositional culture to survive and there is therefore full assimilation, i.e. $q_{m}^{*}=1$.

\footnotetext{
${ }^{12}$ The proofs of all propositions can be found in the Appendix.
} 


\subsection{Full cultural segmentation equilibrium}

An interesting special case to investigate is the situation when there is full cultural segmentation (i.e. $d=0$ ). In such a case, cultural transmission occurs only through the exposure to role models inside the minority community. The dynamic equation of evolution of the oppositional trait now follows the following equation:

$$
q_{m, t+1}-q_{m, t}=\left(1-q_{m, t}\right) q_{m, t}\left(\tau_{m}^{*}-\tau_{o}^{*}\right)
$$

which has the familiar form of a replicator dynamics, except for the fact that the "fitness" values are explicitly derived from a process of cultural socialization and thus reflect the socialization efforts $\tau_{m}^{*}$ and $\tau_{o}^{*}$ of the families. In that case, as there is no "imposed" socialization stemming from the exposition to majority role models, and the long-run fraction of oppositional individuals in the minority community is given by:

$$
\tau_{m}^{*}=\tau_{o}^{*}
$$

Since $d=0$, then, by using (20) and (21), we have that: $1-r_{m}=1-q_{m}$ and $1-r_{o}=q_{m}$. As a result, using (24), equation (29) is equivalent to:

$$
\frac{q_{m}^{*}}{1-q_{m}^{*}}=\frac{\Delta V_{m}\left(U, Q_{m}\left(q_{m}, s, q_{b}\right) I_{m}\right)}{\Delta V_{o}\left(U, Q_{o}\left(q_{m}, s, q_{b}\right) I_{o}\right)}
$$

Simple inspection shows that this equation has a unique interior solution described in Figure 1. It is indeed easy to see that the RHS of (30) is a decreasing function of $q_{m}$ while the LHS of (30) is an increasing function of $q_{m}$, and that the two curves only cross once at $q_{m}^{*}$.

\section{[Insert Figure 1 here]}

\subsection{Comparative statics}

Let us now consider the general case, $d>0$, and analyze the properties of the interior equilibrium described in Proposition 1. We thus assume that (28) holds.

\subsubsection{Prevalence of an oppositional culture}

In the case of an interior solution with an oppositional culture, the model provides interesting comparative statics results on various variables of the environment of the minority group. 


\section{Proposition 2 (Prevalence of an oppositional culture)}

(i) An increase in the opportunity cost $U$ reduces the prevalence of an oppositional culture;

(ii) A reduction of segregation (i.e. an increase in s) increases the prevalence of an oppositional culture;

(iii) An increase in the size of the minority group $q_{b}$ has an ambiguous effect on the prevalence of an oppositional culture;

(iv) A reduction of cultural segmentation (i.e. an increase in d) has an ambiguous effect on the prevalence of an oppositional culture.

Indeed, increasing the opportunity cost $U$ of the action prescribed by the oppositional culture (i.e. action $B$ ) tends to increase the marginal socializing incentives $\Delta V_{m}$ of the mainstream families while it decreases the marginal socializing incentives $\Delta V_{o}$ of the oppositional families. This, in turn, increases the transmission effort $\tau_{m}^{*}$ of mainstream parents and decrease that of oppositional parents, $\tau_{o}^{*}$. As a result, this leads to a lower prevalence of oppositional preferences within the minority group.

Similarly, a reduction in segregation tends to intensify oppositional identities (i.e. increases $\alpha_{o}$ ) while it reduces the identity intensity of mainstream minorities (i.e. reduces $\left.\alpha_{m}\right)$. This, in turn, tends to increase the marginal socializing incentives $\Delta V_{o}$ of the oppositional families while it decreases that of mainstream families, $\Delta V_{m}$. The resulting increase in $\tau_{o}^{*}$ and decrease in $\tau_{m}^{*}$ lead to a rise in the prevalence of oppositional types in the minority group.

It is interesting to scrutinize the ambiguity results of $q_{b}$ and $d$ on $q_{m}^{*}$. There are in fact two opposite effects: a socialization level effect and a marginal socialization effect. Take for instance an increase in $q_{b}$, the size of the minority group. Simple differentiation of the steady state value $q_{m}^{*}$ gives:

$$
\frac{\partial q_{m}^{*}}{\partial q_{b}}=-\frac{1}{\partial \Omega / \partial q_{m}}\{\underbrace{q_{m} d\left(\tau_{m}^{*}-\tau_{o}^{*}\right)-\left(1-\tau_{o}^{*}\right) d}_{\text {Socialization level effect: }-}+\underbrace{q_{m}\left(1-d+d q_{b}\right)\left(\frac{\partial \tau_{m}^{*}}{\partial q_{b}}-\frac{\partial \tau_{o}^{*}}{\partial q_{b}}\right)}_{\text {Marginal socialization effect: }+}\}
$$

A larger size of the minority group $q_{b}$ tends to increase the likelihood for children to be exposed to oppositional role models as they only exist in the minority community. This, in turn, promotes cultural transmission towards the oppositional trait $o$. This is reflected in (31) 
by the first two negative expressions. ${ }^{13}$ At the same time, a larger minority population makes it more likely for minority individuals to socially interact. This increases the probability $Q_{m}$ for a mainstream minority of matching with an oppositional individual while, on the opposite, it tends to reduce the probability $Q_{o}$ for an oppositional person of matching with a mainstream individual since there are no oppositional individuals in the majority group. This leads to an increase in identity intensity $\alpha_{m}$ from the mainstream minorities and a decrease in identity intensity $\alpha_{o}$ from the oppositional minorities. This leads to a larger prevalence of the cultural transmission effort from the mainstream minorities and a reduction in cultural transmission effort from the oppositional minorities. This marginal effect of socialization will favor the prevalence of the mainstream trait inside the minority community. This effect is illustrated by the last positive term in (31).

Again, a particular interesting case in which this ambiguity can be solved is when there is full cultural segmentation (i.e. $d=0$ ). In that case, there is no socialization level effect since minority children are not exposed at all to the majority role models and only the positive marginal socialization effect remains. Thus, we have:

Corollary 1 When there is perfect cultural segmentation (i.e. $d=0$ ), an increase in the size of the minority group reduces the prevalence of an oppositional minority culture, i.e. $\left.\frac{\partial q_{m}^{*}}{\partial q_{b}}\right|_{d=0}<0$.

Similarly, as we have seen in Proposition 2, a change in cultural segmentation $d$ has an ambiguous effect on $q_{m}^{*}$ because of the opposite effects of the socialization level and marginal effects. Indeed, a simple differentiation gives:

$$
\frac{\partial q_{m}^{*}}{\partial d}=-\frac{1}{\partial \Omega / \partial q_{m}}\{\underbrace{\left(1-q_{b}\right)\left[-q_{m}\left(\tau_{m}^{*}-\tau_{o}^{*}\right)+\left(1-\tau_{o}^{*}\right)\right]}_{\text {Socialization level effect: }+}+\underbrace{q_{m}\left(1-d+d q_{b}\right)\left(\frac{\partial \tau_{m}^{*}}{\partial d}-\frac{\partial \tau_{o}^{*}}{\partial d}\right)}_{\text {Marginal socialization effect: }-}\}
$$

Indeed, a reduction in cultural segmentation tends to increase the "imposed" cultural influence of the majority group. This promotes the diffusion of mainstream values inside the minority group. This is the socialization level effect and it is reflected by the two first positive terms in equation (32). On the other hand, there is also a negative marginal socialization effect which is related to the way different families react to the increased exposure to role models of the majority group. Indeed, because family and social models are cultural substitutes, an increased exposure to models of the majority group will reduce the family

\footnotetext{
${ }^{13}$ Remember that, in equilibrium, $\tau_{m}^{*} \leq \tau_{o}^{*}$.
} 
socialization effort of mainstream minority families but will increase that of oppositional families. This marginal effect per se tends to favor the diffusion of oppositional views inside the minority group. This is described by the last negative term in (32).

It is interesting to consider once more the full cultural segregation case when $d=0$. We have the following result:

Corollary 2 When socialization costs of families are convex enough, a reduction of cultural segmentation at the full cultural segmentation equilibrium reduces the prevalence of the oppositional culture.

\subsubsection{Intensity of identity}

The model also provides some comparative statics on identity intensities within the minority group. For any parameter $v \in\left\{U, s, q_{b}, d\right\}$, simple differentiation of (6) gives:

$$
\frac{d \alpha_{i}}{d v}=\frac{d \alpha_{i}}{d Q_{i}}\left[\frac{\partial Q_{i}}{\partial v}+\frac{\partial Q_{i}}{\partial q_{m}} \frac{d q_{m}^{*}}{d v}\right]
$$

which after substitution leads to:

$$
\begin{aligned}
\frac{d \alpha_{m}^{*}}{d v} & =\frac{d \alpha_{m}^{*}}{d Q_{m}}\left[\frac{\partial Q_{m}}{\partial v}-\left(1-s+s q_{b}\right) \frac{d q_{m}^{*}}{d v}\right] \\
\frac{d \alpha_{o}^{*}}{d v} & =\frac{d \alpha_{m}^{*}}{d Q_{o}}\left[\frac{\partial Q_{o}}{\partial v}+\left(1-s+s q_{b}\right) \frac{d q_{m}^{*}}{d v}\right]
\end{aligned}
$$

There are two effects: a short-run effect, evaluated for a given fraction of oppositional individuals $1-q_{m}$ in the minority group, and a long-run effect, taking into account the full dynamic implications on the evolution of the oppositional trait in the group. The short-run effect, reflected in the term $\frac{d \alpha_{i}}{d Q_{i}} \frac{\partial Q_{i}}{\partial v}$, comes from the impact of a change in one parameter on the probability $Q_{i}$ of matching a different cultural type in the population. This has immediate effects on the intensity of identity since individuals tend to vary their identity strength in order to change their psychological cost of such matching on their utility. The long run effect, reflected in the term $\frac{d \alpha_{i}}{d Q_{i}} \frac{\partial Q_{i}}{\partial q} \frac{d q^{*}}{d v}$, stems from the fact that a change in a parameter $v$ may affect as well the long-run frequency of oppositional types in the minority population. This, in turn, has long run implications on the probability of social interactions with individuals who share different values. In particular, if the frequency of oppositional types increases in the long run (i.e. $q_{m}^{*}$ decreases), then this triggers a reaction of intensity of identity of mainstream minority individuals while, on the contrary, it reduces the identity intensity for oppositional families. More specifically: 
Corollary 3 An increase in the opportunity cost $U$ of the oppositional culture has no effect in the short run while, in the long run, it tends to reduce the identity intensity of mainstream individuals and increase it for oppositional individuals in the minority group.

Intuitively, as there are less oppositional persons in the minority community, people tend to match more often with individuals who do not share their views. This, in turn, tends to make them more confident about their own identity. The opposite occurs for mainstream minority individuals.

Consider now a change in $s$, the segregation parameter. A reduction in segregation has now a short run and a long run effects. The short run impact comes from the fact that an increase in $s$ increases $Q_{o}$ and reduces $Q_{m}$. This tends to increase the identity intensity $\alpha_{o}$ of oppositional individuals and reduce the identity intensity $\alpha_{m}$ of mainstream people. At the same time, however, a reduction in segregation increases the prevalence of oppositional families in the minority group as it stimulates their cultural transmission efforts relative to mainstream parents. This increased prevalence of oppositional types leads, in turn, to a decrease of the oppositional identity intensity and to an increase of the mainstream identity. It follows therefore that the long run effect goes in opposite direction to the short-run impact. In general, the full effect of a change in segregation on identity intensity is ambiguous. Again however for situations close to full cultural segmentation (i.e. $d=0$ ), one can resolve this ambiguity. We have:

Corollary 4 Assume $d=0$. Then, a reduction in segregation (i.e. an increase in $s$ ) leads to a positive impact on oppositional identity and a negative impact on mainstream identity. The long run effect is smaller than the short run impact.

\section{Justification of assumptions and modelling choices}

Our model, like most models, is based of assumptions and modeling choices. It is relatively complex since we model three main aspects: $(i)$ the parental investment in transmitting his own trait, $(i i)$ the role of peers and neighborhood when this transmission fails, (iii) the choice of intensity of identity of children.

One could have, for example, developed a simpler model with no parental investment. Let us now argue what we believe that this last aspect is crucial for the result.

First, if there were no parental investment then the dynamics of $q_{m}$ will be trivial (see (27)) since they will be no interior equilibrium. The richness of our model is the fact that both $\tau_{m}^{*}$ and $\tau_{o}^{*}$ are choice variables and depend on $q_{m}, q_{b}, s, d$, and $U$. 
Second, how important is parental investment in transmitting ethnic identity in the real world? Observe that we are not looking at the transmission of a trait for which everybody agrees on the fact that it is good or bad (like, for example, education or crime). In that case, no parent will put effort (which is costly) in transmitting "bad" education or criminal behavior. This is not what we are modelling here. We are looking at ethnic identity issues and how ethnic identity is transmitted from one generation to another. Identity is horizontally differentiated (i.e. it is a matter of taste which trait one wants to transmit) while crime or bad behavior is vertically differentiated (i.e. all parents agree that crime is not good and thus put no effort in transmitting this trait). In that respect, we believe that the assumption of imperfect empathy (or paternalistic altruism) makes sense in this framework. ${ }^{14}$ All parents, especially those with a strong ethnic identity, are biased in their evaluation of the wellbeing of their offsprings. Otherwise, how can we understand that some parents prefer their daughter not to go to school rather than not wearing the Islamic veil (there were a famous case in England some years ago). Also, why parents in some communities are actively putting effort for arranged marriages for their kids if not for keeping a strong identity. Arranged marriage is still common in South America, India, Pakistan, Japan, Iran, etc. Among most Indian and Nepalese Hindus, the hereditary system of caste is an extremely important factor in arranged marriage. Arranged marriages, and parents, almost always require that the married persons should be of the same caste. Couples who defy arranged marriages in certain (especially rural) places, are sometimes separated, ostracized, or even killed. In that case, parents clearly evaluate the well-being of their children as if it was their own utility.

So what about the evidence on parental transmission of ethnic identity. In the psychology/sociology literature, the family is considered the primary socializing agent of the group because it is the first institution with which an individual comes into contact, and often the last institution with which the individual has final ties (Johnson, 1981). In comparison to other influences, family socialization has been said to have the most influential and lasting impact on the child's competencies as a functioning human being (Harrison et al, 1990). One aspect of family socialization, ethnic socialization, entails preparing the child for different environmental niches by giving the child a positive sense of ethnic identity (Boykin, 1986; Johnson, 1981). The family, by inculcating a positive sense of ethnic identity (Hughes and Demo, 1989) serves as a buffer from the impact of the child's minority status (Jackson et al., 1981).

One of the first empirical examinations of ethnic socialization among African American parents was conducted by Marie Ferguson Peters. Peters (1985), in her Toddler Infant Expe-

\footnotetext{
${ }^{14}$ See Bisin and Verdier (2000) page 962 for evidence on the "imperfect empathy" assumption.
} 
riences Study (TIES), examined African American parents' socialization of their preschoolers. The global findings from her empirical, descriptive study suggest that the African American mothers were gravely concerned about their children growing up in an environment that was generally not safe for them because of their ethnicity. Additionally, the parents tended to emphasize the following in their socialization practices: (1) self-esteem, (2) positive feelings about their ethnicity, (3) self-respect, (4) lack of fair and honest treatment from White Americans, and (5) education.

Another study that addresses this issue is that conducted by Demo and Hughes (1990). In their study, utilizing the National Survey of Black Americans data set, these researchers examined the relationship of racial identity to ethnic socialization. In distinguishing between the different types of socialization strategies employed by parents, the authors report that parents who took an assertive/integrative style to socializing their children had children who felt a greater attachment to their ethnic group. An assertive/integrative style is one in which the parent instructed the child on the general importance of their black heritage, the equality of all people, and the importance of getting along with white people. Thus, racial group identity as measured through the individual's feeling of closeness to other blacks was impacted by the parent's emphasis on preparing the child for a race-conscious milieu.

Spencer (1983) investigated the relationship between the socialization practices of black parents and their children's feelings and preferences about black culture. Spencer found that parents who talk to their children about the civil rights movement, black history, and discrimination had children who expressed a more positive attitude about black culture than did the children of parents who did not discuss these issues.

Focussing on the Hispanic ethnic-identity transmission, Knight et al. (1993) show that parents communicate ethnic content to their children through such means as child-rearing practices, teaching, media. Mothers who taught about the Mexican culture, pride and discrimination had children who engaged in more ethnic behaviors, who used more Mexican behaviors and expressed more ethnic preferences. They conclude that mothers who teach and model their children about the Mexican culture have effects on their children ethnic identity.

Investigating the transmission of Jewish ethnic identity, Davey et al. (2003) find that clear expectations, a type of authoritative parenting, could style be associated with the positive transmission of Jewish ethnic identity. This type of parenting style was direct as parents expressed clear expectations for participation in Jewish activities both at home and in the community.

All this evidence points out to the fact that parental investment in transmitting ethnic 
identity is crucial to understand the emergence of ethnic identity among their children.

\section{Harassment from the dominant group}

\subsection{Unconditional harassment}

We would like now to extend the previous model to analyze the impact of racial harassment on the existence and evolution of a oppositional minority culture. Let us assume that, in the majority group, there are some individuals who are negatively affected when matched with a minority individual (it does not matter if this minority person is mainstream or oppositional). Because they feel a loss of identity $I_{\gamma}$ when matched with a minority person, they are ready to take an harassment action $Z$ in order to recover part of their identity loss (i.e. reduce the identity cost). We refer to these individuals as racists and we denote by $\gamma$ the fraction of racist individuals in the majority group. We assume that the racist individuals have to pay a cost $c Z$ for the harassment action $Z$, which consequently reduces their identity cost to $I_{\gamma}=I_{\gamma}(Z)$ with $I_{\gamma}^{\prime}(Z)<0$ and $I_{\gamma}^{\prime \prime}(Z) \geq 0$. After matching with a minority individual, the harassment level chosen by a racist person will then be determined by the following program:

$$
\max _{Z}\left\{-I_{\gamma}(Z)-c Z\right\}
$$

The first order condition gives:

$$
-I_{\gamma}^{\prime}\left(Z^{*}\right)=c
$$

which defines an optimal harassment level $Z^{*}=Z(c)$, with $Z^{\prime}(c) \leq 0$ since higher costs reduces the level of harassment chosen.

We assume that the harassment $Z$ against a minority individual has two effects. First, it negatively affects the expected economic payoff of action $G$ for an ethnic minority. Second, it increases the psychological cost $I_{o}$ for a oppositional person of interacting with a mainstream individual from the majority group. Therefore, $U$, the economic returns of action $G$, is now a function of $Z$, i.e. $U=U(Z)$, with $U^{\prime}(Z)<0$ and $U^{\prime \prime}(Z) \leq 0$, and $I_{o}$, the psychological cost for an oppositional individual of interacting with a mainstream individual, is also a function of $Z$, i.e. $I_{o}=I_{o}(Z)$ with $I_{o}^{\prime}(Z)>0$ and $I_{o}^{\prime \prime}(Z) \geq 0$. As a result, contrary to (1) and (2), the economic returns of action $G$ now depends with whom the minority individual is matched. As a result, the expected payoff of undertaking action $G$ is now given by:

$$
E U(Z, \gamma)=s\left(1-q_{b}\right) \gamma U(Z)+\left[1-s\left(1-q_{b}\right) \gamma\right] U(0)
$$


Indeed, with probability $s\left(1-q_{b}\right) \gamma$, a minority individual is matched with a racist majority individual and therefore suffers the socio-economic cost of harassment, i.e. $U(Z)$. With the residual probability, he is not matched to a racist person and enjoys a full payoff $U(0)=U \cdot{ }^{15}$

We are now able to solve the choice of identity intensity. Let us start with a mainstream minority individual. He will solve the following program:

$$
\max _{\alpha_{m}}\left[E U(Z, \gamma)-\lambda\left(\alpha_{m}\right) Q_{m} I_{m}-C\left(\alpha_{m}\right)\right]
$$

Observe that, when choosing optimally $\alpha_{m}$, this mainstream person will not be affected by harassment $Z$ because $E U(Z, \gamma)$ does not depend on $\alpha_{m}$ and there is an expected psychological cost only if one interacts with someone with a different trait. Since here the person is mainstream, the only cost $I_{m}$ borne is when he interacts with an oppositional person. Since by definition there is no oppositional individuals within the majority group, the mainstream minority individual will not suffer any identity cost associated to harassment. So basically, when a mainstream minority meets a racist from the majority group, he suffers a socioeconomic loss, which is increasing with the level of harassment $Z$ (as $U(Z)$ is decreasing with $Z$ ). He bears, however, no psychological costs since, in terms of identity, his values are similar to that of a mainstream (even racist) individual. As a result, the solution of this program is still given by (6) and $\alpha_{m}^{*}$ is not a function of $Z$ or $\gamma$.

On the contrary, for an oppositional individual, the program to be solved is not anymore given by (5) but by:

$$
\max _{\alpha_{o}}\left[-\lambda\left(\alpha_{o}\right)\left\{s\left(1-q_{b}\right) \gamma I_{o}(Z)+\left[\left(1-s+s q_{b}\right) q_{m}+s\left(1-q_{b}\right)(1-\gamma)\right] I_{o}\right\}-C\left(\alpha_{o}\right)\right]
$$

Indeed, when someone is oppositional there is a cost of choosing action $B$. If this person meets a racist (which occurs with probability $s\left(1-q_{b}\right) \gamma$ ), the cost is $I_{o}(Z)$. If this oppositional individual does not meet a racist majority individual, he can either meet a minority worker (this occurs with probability $\left(1-s+s q_{b}\right) q_{m}$ ) or a majority individual who is not racist (this occurs with probability $\left.s\left(1-q_{b}\right)(1-\gamma)\right)$ and obtain in both cases a fixed cost of $I_{o}(0) \equiv I_{o}$. As a result, the term inside the curly bracket is simply the expected loss of identity of such an oppositional individual.

The optimal level of identity intensity for an oppositional individual is then given by the following first order condition:

$$
C^{\prime}\left(\alpha_{o}^{*}\right)=-\lambda^{\prime}\left(\alpha_{o}^{*}\right)\left\{s\left(1-q_{b}\right) \gamma I_{o}(Z)+\left[\left(1-s+s q_{b}\right) q_{m}+s\left(1-q_{b}\right)(1-\gamma)\right] I_{o}\right\}
$$

\footnotetext{
${ }^{15}$ We assume that $E U(Z, \gamma)>0, \forall \gamma \in[0,1]$ so that mainstream minority individuals always choose action $B$ as their optimal action.
} 
reflecting the fact that this individual only suffers a higher identity loss when he interacts with a majority individual that harasses him. We denote the solution of this equation by $\alpha_{o}^{*}(Z, \gamma)$. We can now calculate the marginal incentives of cultural transmission for parents of the two types $m$ and $o$. They are given by:

$$
\begin{gathered}
\Delta V_{m}(Z, \gamma)=E U(Z, \gamma)+\Psi\left(\alpha_{m}^{*}\right) \\
\Delta V_{o}(Z, \gamma)=\Phi\left(\alpha_{o}^{*}(Z, \gamma)\right)-E U(Z, \gamma)
\end{gathered}
$$

where $\Delta V_{m}(Z, \gamma)$ is decreasing in both $Z$ and $\gamma$ while $\Delta V_{o}(Z, \gamma)$ is increasing in both $Z$ and $\gamma$. It follows that the cultural transmission effort $\tau_{m}^{*}\left(q_{m}, Z, \gamma\right)$ of a mainstream minority parent is shifted downward by $Z$ and $\gamma$. Conversely, the oppositional cultural transmission effort $\tau_{o}^{*}\left(q_{m}, Z, \gamma\right)$ is shifted upward by $Z$ and $\gamma$. As a result, the interior steady-state of cultural dynamics is the solution of the following equation:

$$
q_{m}\left[1-d+d q_{b}\right]\left[\tau_{m}^{*}\left(q_{m}, Z, \gamma\right)-\tau_{o}^{*}\left(q_{m}, Z, \gamma\right)\right]+\left[1-\tau_{o}^{*}\left(q_{m}, Z, \gamma\right)\right] d\left(1-q_{b}\right)=0
$$

This occurs once again when:

$$
\Psi^{\prime}\left(d\left(1-q_{b}\right)\right)<\Phi\left(\alpha_{o}^{*}(Z, \gamma)\right)-E U(Z, \gamma)
$$

Note that it is now the right-hand side of this inequality that is increasing in $Z$ and $\gamma$ and we have therefore this straightforward result:

Proposition 3 (Oppositional culture and unconditional harassment) An oppositional minority culture is more likely to emerge the higher the level of harassment $Z$ and the higher the number of racist individuals in the society.

As already mentioned in Proposition 2, an increase in social integration (i.e. an increase in $s$ ) increases the likelihood of the emergence/persistence of an oppositional minority culture. It should be noted as well that social integration and harassment by members of the majority group tend to be complementary in stimulating the emergence of an oppositional minority culture. As a matter of fact, under mild technical conditions, it can be shown that the right-hand side $\Psi=\Phi\left(\alpha_{o}^{*}(Z, \gamma)\right)-E U(Z, \gamma)$ of inequality (35) is increasing in $Z$ and $\gamma$, at an increasing rate in $s$ (i.e. formally $\frac{\partial^{2} \Psi}{\partial s \partial Z}>0$ and $\frac{\partial^{2} \Psi}{\partial s \partial \gamma}>0$ ). ${ }^{16}$ Therefore, the following conclusion can be drawn:

\footnotetext{
${ }^{16}$ Indeed, it is easy to see through differentation of (33) that $\frac{\partial^{2} E U}{\partial s \partial \gamma}<0$ and $\frac{\partial^{2} E U}{\partial s \partial Z}<0$. Also when $\Phi(\alpha)$ is sufficiently $\log$ convex (i.e. $\frac{\Phi^{\prime \prime}}{\Phi^{\prime}}>\frac{\Sigma^{\prime \prime}}{\Sigma^{\prime}}$ where $\left.\Sigma(\alpha)=\frac{C^{\prime}(\alpha)}{-\lambda(\alpha)}\right)$, using as well (34), it can also be shown that $\frac{\partial^{2} \Phi\left(\alpha_{o}^{*}\right)}{\partial s \partial \gamma}>0$ and $\frac{\partial^{2} \Phi\left(\alpha_{o}^{*}\right)}{\partial s \partial Z}>0$. From this, one will get $\frac{\partial^{2} \Psi}{\partial s \partial Z}>0$ and $\frac{\partial^{2} \Psi}{\partial s \partial \gamma}>0$.
} 
Corollary 5 An increased level of harassment and a higher number of racist individuals in the majority group is more likely to promote the emergence of an oppositional minority culture, the less socially segmented is the minority group.

The intuition is quite simple. More social interactions with the majority group induces more chance to suffer harassment and racism from that group. This reduces the economic return to adopt the mainstream culture as well as it reinforces the identity of being an oppositional type. The two channels favor the existence of an oppositional minority culture.

\subsection{Conditional harassment}

Assume now that harassment by the majority group is conditional on non-integration or opposition to the dominant culture from ethnic minorities. More precisely, consider the case where the racists feel an identity loss only against a minority individual if the latter is oppositional. In other words, this is not as before pure racism against the minority group but rather a form of conditional racism, based on fear and resentment of facing minority individuals not conforming to mainstream values. Assume as well that the oppositional type is not directly observable by a majority individual when socially interacting with that person. ${ }^{17}$ Hence, after matching with a minority type, the "conditional" racist faces now the following problem:

$$
\max _{Z}\left\{-\left(1-q_{m}\right) I_{\gamma}(Z)-c Z\right\}
$$

in which he minimizes his expected loss of identity when matched with a minority individual. This program gives a solution $Z\left(q_{m}, c\right)$, which is decreasing in $q_{m}$, with $Z(1, c)=0$. The level of harassment is now frequency dependent and increasing in the proportion $1-q_{m}$ of oppositional individuals. The oppositional individuals are imposing therefore a negative externality on the mainstream minority individuals.

An interior cultural steady state equilibrium is now the solution of :

$$
q_{m}\left(1-d+d q_{b}\right)\left[\tau_{m}^{*}\left(q_{m}, Z, \gamma\right)-\tau_{o}^{*}\left(q_{m}, Z, \gamma\right)\right]+\left[1-\tau_{o}^{*}\left(q_{m}, Z, \gamma\right)\right] d\left(1-q_{b}\right)=0
$$

\footnotetext{
${ }^{17} \mathrm{An}$ alternative less extreme assumption would be that the oppositional type can be partially inferred from external signals (like clothes, physical appearance, manners, etc...). As long as the signal provides an ex-post probability of having an oppositional type that is increasing in the fraction $1-q_{m}$ of oppositional individuals within the minority group, the results will be qualitatively the same.
} 
with $Z=Z\left(q_{m}, c\right)$. Solving this equation gives $q_{m}^{*}=q_{m}(Z, \gamma)$, which we know to be decreasing in $Z$ and $\gamma$. Therefore, we need to solve:

$$
q_{m}^{*}=q_{m}(Z, \gamma) \text { and } Z^{*}=Z\left(q_{m}, c\right)
$$

Again, there is at least one steady state as illustrated in Figures 2a and 2b.

$$
\text { [Insert Figures } 2 a \text { and } 2 b \text { here] }
$$

However, there might be now more than one steady-state equilibrium. Because of the externality that oppositional individuals are exerting on harassment, a larger number of oppositional types are creating more social harassment. In particular, action $B$ has a lower return, while oppositional individuals tend to become more polarized in terms of their identity intensity. Both dimensions, in turn, tend to reduce the cultural success of mainstream preferences inside the minority group and to further stimulate the diffusion of oppositional values over time inside that community. Harassment and oppositional culture exert on each other complementarities that may generate multiple steady states of cultural values in the minority group. When there is more than one equilibrium, as shown for instance in Figure $2 \mathrm{~b}$, one has at least one low equilibrium with little harassment and a low prevalence of oppositional minority individuals $\left(q_{m L}^{*}, Z_{L}^{*}\right)$ and a high equilibrium with high harassment and a larger presence of oppositional individuals $\left(q_{m H}^{*}, Z_{H}^{*}\right)$. At any stable steady state $q_{m}^{*}$ and $Z^{*}$, it is straightforward to obtain the following comparative statics results:

Proposition 4 (Oppositional culture and conditional racists) The prevalence of an oppositional minority culture is more likely to arise the higher the number $\gamma$ of "conditional" racists in the society and the lower the cost $c$ of the harassment. The equilibrium level of harassment $Z^{*}$ is increasing in the number of "conditional" racists and it decreasing in both the cost $c$ and the level of segregation (i.e. higher s) between minority and majority individuals.

\section{Evolution of intolerance and oppositional cultures}

So far, we assumed that the fraction of individuals with "racist preferences" in the majority group was exogenous. Still, sociologists have long argued that racial prejudices and discrimination in the dominant group tend to be endogenous and to increase with the size of the subordinate group (see e.g. Blatock 1956, 1957, 1967). For instance, using data from the Eurobarometer Survey on individual attitudes towards immigrants and racial minorities 
across 12 countries, Quillian (1995), controlling for individuals factors, shows the existence of a positive correlation between population size of the racial minority and the degree of racial prejudices expressed by natives of the country. This literature suggests that "racist preferences" against minority groups are actually endogenous to the pattern of assimilation of these minority groups. In our model, this feature could be captured by endogenizing $\gamma$, the fraction of racist individuals. This is what is done now and we find it useful to distinguish between "conditional" and "unconditional" racists.

\section{1 "Conditional" racists}

Assume that the trait "racism" among the majority group is transmitted from one generation to another through a mechanism that interacts cultural transmission and socialization inside the family, and social interactions and peer effects, via imitation and learning. This is the way we modelled the transmission of the traits "oppositional" and "mainstream" in the minority group in this paper. There are now two cultural traits in the majority group: the trait $N R$ "non racist", according to which there is no identity loss when matching with a minority individual, and the trait $R$ "racist", which generates identity aversion only against oppositional minority individuals (in the previous section, we called these individuals "conditional" racist). Individuals $N R$ do not undertake any harassment action and thus do not pay the harassment cost $c Z$. Concerning individuals $R$, without loss of generality, we assume that the harassment decision is discrete $\{0, Z\}$, with an harassment cost $c Z$ for action $Z>0$. Let us denote by $\Delta I_{\gamma}=I_{\gamma}(0)-I_{\gamma}(Z)$, the "racist" identity gain from harassing an oppositional minority individual, with $\Delta I_{\gamma}>c Z$. As before, the utility of a person from the majority group is: $-\left(1-q_{m}\right) I_{\gamma}(Z)-c Z$, if he chooses to harass an oppositional minority individual, and $-\left(1-q_{m}\right) I_{\gamma}(0)$, if not. As a result, when minimizing their expected identity loss, "conditional" racists will now take an harassment decision according to the following rule:

$$
\text { Choose }\left\{\begin{array}{rrr}
Z & \text { when } \quad q_{m} \leq 1-c Z / \Delta I_{\gamma} \\
0 & \text { when } \quad q_{m}>1-c Z / \Delta I_{\gamma}
\end{array}\right.
$$

When the fraction of oppositional minority individuals is large enough (i.e. $q_{m}$ small enough), then majority individuals choose the harassment action $Z>0$. In this context, the cultural evolution of the fraction $\gamma$ of "conditional" racists is simply given by:

$$
\gamma_{t+1}-\gamma_{t}=\gamma_{t}\left(1-\gamma_{t}\right)\left(\tau_{R}-\tau_{N R}\right)
$$


where the cultural transmission efforts $\tau_{R}$ and $\tau_{N R}$ of "conditional" racist and non racist families are determined by:

$$
\Theta^{\prime}\left(\tau_{R}\right)=\left(1-\gamma_{t}\right) \Delta V_{R}\left(q_{m, t}\right) \text { and } \Theta^{\prime}\left(\tau_{N R}\right)=\gamma_{t} \Delta V_{N R}\left(q_{m, t}\right)
$$

and where the marginal incentives of cultural transmission $\Delta V^{R}\left(q_{m, t}\right)$ and $\Delta V^{N R}\left(q_{m, t}\right)$ are given by:

$$
\Delta V_{R}\left(q_{m, t}\right)=\left\{\begin{array}{ccc}
\left(1-q_{m, t}\right) \Delta I_{\gamma}-c Z & \text { when } & q_{m, t} \leq 1-c Z / \Delta I_{\gamma} \\
0 & \text { when } & q_{m, t}>1-c Z / \Delta I_{\gamma}
\end{array}\right.
$$

and

$$
\Delta V_{N R}\left(q_{m, t}\right)=\left\{\begin{array}{ccl}
c Z & \text { when } \quad q_{m, t} \leq 1-c Z / \Delta I_{\gamma} \\
0 & \text { when } \quad q_{m, t}>1-c Z / \Delta I_{\gamma}
\end{array}\right.
$$

Note that $\Delta V_{R}\left(q_{m, t}\right)$ and $\Delta V_{N R}\left(q_{m, t}\right)$ differ from each other only when the two preferences $R$ and $N R$ induce a different behavior with respect to minority individuals (i.e. when $\left.q_{m, t} \leq 1-c Z / \Delta I_{\gamma}\right)$. The full dynamic system of cultural evolution in the two variables $\left\{q_{m, t}, \gamma_{t}\right\}$ can then be written as:

$$
\begin{gathered}
q_{m, t+1}-q_{m, t}=\left(1-q_{m, t}\right)\left\{q_{m, t}\left[\tau_{m}\left(q_{m, t}, Z, \gamma_{t}\right)-\tau_{o}\left(q_{m, t}, Z, \gamma_{t}\right)\right]+\left[1-\tau_{o}\left(q_{m, t}, Z, \gamma_{t}\right)\right] d\left(1-q_{b}\right)\right\} \\
\gamma_{t+1}-\gamma_{t}=\gamma_{t}\left(1-\gamma_{t}\right)\left(\tau_{R}-\tau_{N R}\right)
\end{gathered}
$$

The interior steady states $\left(q_{m C}^{*}, \gamma_{C}^{*}\right)$ are given by the following equations: ${ }^{18}$

$$
q_{m C}^{*}=q_{m C}^{*}\left(Z, \gamma_{C}\right) \text { and } \gamma_{C}^{*}\left(q_{m C}\right)=\frac{\Delta V_{R}}{\Delta V_{R}+\Delta V_{N R}}=1-\frac{c Z}{\Delta I_{\gamma}\left(1-q_{m C}\right)}
$$

It is convenient to restrict the discussion to the case when $q_{m C}^{*}(Z, 0) \leq 1-c Z / \Delta I_{\gamma}$, namely when there are positive incentives for harassment by the first marginal "conditional" racist when the minority population is at the steady state value with no "conditional" racists. This is a situation in which "conditional" racist preferences can initially culturally invade a majority with no-racist preferences. The phase diagram is then described by Figures $3 \mathrm{a}$ and $3 b$.

\section{[Insert Figures $3 a$ and $3 b$ here]}

In principle, there might be multiple steady states, alternating stable and unstable interior steady states. It is immediate to obtain the following result

\footnotetext{
${ }^{18}$ Subscript $C$ refers to the case of "Conditional" racists.
} 


\section{Proposition 5 (Oppositional culture and segmentation)}

(i) At a stable interior steady-state equilibrium $\left(q_{m C}^{*}, \gamma_{C}^{*}\right)$, the prevalence of an oppositional minority culture is more likely to arise, the lower the level of segregation in the society, and the lower the cost of harassment. Also, at $\left(q_{m C}^{*}, \gamma_{C}^{*}\right)$, the number of "conditional" racists decreases with segregation and with the cost of harassment.

(ii) In the case of full cultural segmentation (i.e. $d=0$ ), the prevalence of an oppositional minority culture is more likely to arise, the lower the percentage of ethnic minorities in the society while the number of "conditional" racists decreases with the percentage of ethnic minorities in the society

\section{2 "Unconditional" racists}

If now racist preferences are explicitly against any minority member (and not only against oppositional minority individuals), then majority individuals endowed with such preferences will always undertake the harassment action $Z$ when matched with a minority person. The previous model of cultural evolution fully applies, except that the marginal incentives of cultural transmission inside the majority group are modified in the following way:

$$
\Delta V_{R}\left(q_{m, t}\right)=\Delta I_{\gamma}-c Z \text { and } \Delta V_{N R}\left(q_{m, t}\right)=c Z
$$

As can be seen, these marginal incentives do not depend anymore on the frequency $1-q_{m}$ of oppositional individuals in the minority group. It is then immediate to see that the interior steady state is given by the following equations: ${ }^{19}$

$$
q_{m N C}^{*}=q_{m N C}^{*}\left(Z, \gamma_{N C}^{*}\right) \text { and } \gamma_{N C}^{*}=1-\frac{c Z}{\Delta I_{\gamma}}
$$

It is straightforward to show that

$$
q_{m N C}^{*}<q_{m C}^{*} \text { and } \gamma_{C}^{*}<\gamma_{N C}^{*}
$$

There is indeed more cultural polarization (i.e. more oppositional minority individuals and more racist individuals) between the majority and the minority groups when racist preferences of the majority group are unconditionally against minority individuals than when they are only against oppositional minority individuals.

\footnotetext{
${ }^{19}$ The subscript $N C$ refers to the case of "Non-Conditional" racists.
} 


\section{Discussion of the results: Empirical and policy im- plications}

\subsection{Our main results}

By developing a dynamic model that interacts cultural transmission and socialization inside the family, peer effects and social interactions, and identity choice, we have found the following main results:

(i) The prevalence of an oppositional culture in the minority group can be sustained if and only if there is enough cultural segmentation in terms of role models ( $d$ small enough), and/or the size of minority group is large enough ( $q_{b}$ large enough), and/or the degree of oppositional identity it implies is high enough (i.e. $\Phi($.$) and I_{o}$ high enough), and/or the socio-economic opportunity cost of the actions it prescribes is small enough (i.e. $U$ small enough). Also in this steady-state equilibrium, the oppositional minority families exert more cultural transmission effort than the mainstream minority families (Proposition 1);

(ii) The lower is the segregation (i.e. higher $s$ ) and/or the lower is the cultural segmentation (i.e. higher $d$ ) between the minority and majority groups, the higher (resp. the lower) is the socialization effort of oppositional (resp. mainstream) parents (equations (25) and (26)). Furthermore, a higher $s$ implies a higher prevalence of an oppositional culture while the effect is ambiguous for $d$ (Proposition 2 and Corollary 4);

(iii) The intensity of identity to an oppositional culture is stronger, the larger the socioeconomic opportunity cost $U$ of the actions it prescribes. Also, at least close to full cultural segmentation (i.e. $d \simeq 0$ ), an increase in social integration (i.e. higher $s$ ) leads to a positive impact on oppositional identity intensity (Corollary 3)).

(iv) The higher the level of harassment and the higher the number of racist individuals in the society, the more likely an oppositional minority culture emerges (Proposition 3) and even more so in an environment with less social segregation (i.e. higher $s$ ) (Corollary 5). Moreover, the level of harassment is decreasing in the segregation level, i.e. it is increasing in $s$ (Proposition 4 and 5).

$(v)$ Finally, the diffusion of "racist preferences" inside the majority group and the evolution of oppositional culture within the minority group are likely to be dynamic complements, giving rise to multiplier effects of changes of socio-economic parameters, and possibly to the existence of multiple steady-state situations of assimilation and racism in society. 


\subsection{Empirical relevance}

One of our key (policy-relevant) results is to show that desegregation may potentially backfire in some instances, because they increase social interactions across types, and thus can strengthen oppositional identities. This is an important result, which is strongly supported by empirical evidence.

Indeed, the results from a study conducted by Thornton et al. (1990) reveal that structural characteristics influence the propensity of African American parents to engage in ethnic socialization. African American parents who were older, more educated, residing in the Northeast, and who were married were more likely to engage in ethnic socialization. Additionally, those who lived in neighborhoods that were predominantly black were less likely to socialize their children than those who lived in neighborhoods where half of the residents were white. Hence, the greater the number of white people in the neighborhood the more likely the parents were to engage in ethnic socialization (Thornton et al., 1990). ${ }^{20}$

Bisin et al. (2004) study the transmission of religion and evaluate the empirical relevance of the dependence of marriage choices on the distribution of the population by religious group. If our model is correct, this would imply that homogamy will be more prevalent in neighborhoods where the religion in question is less prevalent. They estimate this idea using U.S. survey data, over the period 1972-1996, and simulate the dynamics of the distribution of the population by religious group. Their results suggest that the dependence of marriage rates on the distribution of the population by religious trait displays substantial nonlinearities. Once such nonlinearities are taken into account, they find that ethnic minorities do, in fact, segregate in marriage more intensely than majorities, and they socialize their children more strictly. The observed marriage and socialization patterns are consistent with a strong preference by members of each religious group for having children who share their own religious trait. They also show that when a group is a minority, marriage segregation and socialization efforts are increasing in the group's population share. The reason is that the estimated costs of socialization and marriage segregation are substantial for a minority. As a group grows toward being a majority, marriage segregation and socialization efforts become decreasing in the group's population share. The reason is that when a group population share is high, social interactions favor homogamy and socialization, independent of the explicit effort of

\footnotetext{
${ }^{20}$ Anthropologists have also observed that social groups seek to preserve their identity, an activity that accelerates when threats to internal cohesion intensify. Thus, groups may try to reinforce their identity by penalizing members for differentiating themselves from the group. The penalties are likely to increase whenever the threats to group cohesion intensify; for an early analysis of this issues, see Whyte (1943).
} 
individuals and parents. $^{21}$

More recently, using data on American teenagers, Fryer and Torelli (2010) test the "acting white" phenomenon. They show that for white kids, the higher is the grade, the more popular (in terms of the number of same-race friends) they are while, for black kids, this is true up to a certain grade (3.48). A black student with a 4.0 grade point average has, on average, 1.5 fewer same-race friends than a white student with a 4.0. Another interesting result, in line with the predictions of our model, is that black students in mixed-race schools (a good proxy for neighborhoods) have a stronger identity (in terms of rejecting other blacks with good grades) than in more segregated schools. In other words, racial differences in the relationship between popularity and academic achievement are larger in predominantly white schools relative to predominantly black ones.

Finally, using a unique UK dataset, the Fourth National Survey of Ethnic Minorities, Bisin et al. (2010), find that ethnic identity appears to be formed in social contexts in which the minority ethnic trait is mostly "threatened" either directly by the actions of the majority group (e.g., through explicit acts of rejection or harassment), or indirectly simply by being exposed to the interaction with the majority norm of behavior in mixed neighborhoods.

\subsection{Policy implications}

Let us now discuss these results in terms of policy implications. If the objective of the government is to reduce oppositional identity behaviors in society because it creates tensions between different communities, our model predicts that it should reduce social segregation (i.e. increase $s$ ), cultural segmentation (i.e. increase $d$ ) and better integrate ethnic communities socio-economically (i.e. increase the opportunity cost $U$ of "deviant" behavior).

To reduce segregation, or, equivalently, to induce ethnic minorities to meet people from the majority group, one could promote social mixing. Such policies, which have been implemented in the United States, include school busing, forced integration of public housing, laws barring discrimination in housing and employment, and Moving to Opportunity (MTO) programs, which relocates families from high- to low-poverty neighborhoods (and from racially segregated to mixed neighborhoods). To reduce cultural segmentation, one could increase mainstream role models among the minority group. In that case, positive discrimination or Affirmative Action could be an appropriate policy. ${ }^{22}$

\footnotetext{
${ }^{21}$ Relatedly, Bisin and Verdier (2000) provide many examples of the resilience of ethnic and other cultural traits that can be explained by a similar mechanism, from the case of Orthodox Jews in Brooklyn to the case of aristocrats in France.

${ }^{22}$ See Lang (2007), which gives a very nice overview of these policies in the United States.
} 
Our results $(i),(i i)$ and (iii) indicate that these policies are not equivalent and may not always give the desired results in terms of integration of minorities. Typically, result $(i)$ suggests indeed that less segmentation in terms of cultural socialization (higher $d$ ) and better socio-economic integration (larger $U$ ) are likely to reduce the prevalence of oppositional identity behaviors. On the other hand, result (ii) indicates that, when oppositional identity parents are socially mixed with mainstream individuals (from the minority and majority groups), they may overreact and put much more effort in transmitting their oppositional trait. This, in turn, may lead to more rather than less prevalence of oppositional behaviors.

As well, result (iii) points out to a possible "quantity-quality" trade-off between the prevalence of an oppositional behavior on one side, and, on the other side, the identity intensity attached to this trait. For instance, while better socio-economic integration (larger $U)$ reduces the prevalence of an oppositional cultural trait, it also tends to increase the identity intensity attached to that trait, creating therefore more polarization between existing cultural identities in the society. If identity polarization and extremism as such induce additional social costs, this would have to be weighted against a lower frequency of such oppositional behaviors in the population.

Results $(i v)$ and $(v)$ also show that, by promoting social mixing, some people from the majority group may increase their harassment against the minority group. ${ }^{23}$ This reaction, associated with an increased effort of oppositional parents in transmitting their own trait, could explain why the integration policies cited above have often had limited effects and have been opposed by the same minority groups (see e.g., Jacoby, 1998). For instance, James Coleman, fifteen years after the famous Coleman Report in 1966, which originally proposed busing, admitted that, "the assumption that busing would improve achievement of lower-class black children has now been shown to be a fiction;" (cited in Jacoby, 1999). ${ }^{24}$ Affirmative Action policies (Holzer and Newmark, 2000, 2006) as well as MTO programs (Ludwig et al., 2001, and Kling et al., 2005) have had positive but arguably small effects. ${ }^{25}$ Of course, other aspects could explain why these policies did not work. For example, since the MTO programs basically moved families from extremely poor neighborhoods to poor neighborhoods (Quigley and Raphael, 2008) and involved separating families from their networks of friends (De Souza Briggs et al., 2010), the small effects of these programs say nothing

\footnotetext{
${ }^{23}$ This is supported by empirical evidence. See, in particular, Dustmann and Preston (2001), Rivera-Batiz et al. (2002), Bowyer (2009) and Dustmann et al. (2010).

${ }^{24}$ The failure of the busing and other civil right policies is certainly also due to the whites' flight from de-segregated schools and neighborhoods.

${ }^{25}$ Similarly, the Toronto housing program where adults were assigned as children to different residential housing projects (Oreopoulos, 2003) did not give the expected results in terms of education outcomes.
} 
about lack of benefits from integration. Also, as pointed out by Loury (1995), Affirmative Action programs inherently portray blacks as victims, and can thus encourage oppositional identities.

Two other interesting policies, both highlighted in Austen-Smith and Fryer (2005, pp. 570-571) and Akerlof and Kranton (2010, pp. 106-107), are the US government programs Job Corps and Jobstart. Job Corps is a residential, education and training program for at risk youth, ages 16 to 24 . The important aspect of this program is that it takes the students to training centers where they receive free room and board along with an intense training program. The Job Start is a similar program with one major difference: it is nonresidential and thus students stay at home and commute to a local training site. Job Corps had large effects by increasing earnings and reducing crime while Job Start showed nearly no significant effects. This indicates that neighborhood effects matter. From our model, this could indicate that by taking away minorities from their initial neighborhood and putting them in a more mixed neighborhood could positively affect their outcomes.

Conversely, results $(i v)$ and $(v)$ also emphasize, in the debate on immigrants' cultural assimilation and integration, the importance of dynamic complementarities between, on the one hand, the incentives for minority individuals to adopt mainstream value systems ("the demand side of assimilation") and, on the other hand, the propensity for people from the majority group to feel secure and tolerate without prejudice and discrimination the presence of minority groups around them (the "supply side of assimilation"). The fact that both dimensions are closely inter-related has implications for the design and evaluation of public policies in this area. Indeed, the aforementioned complementarities suggest that a shift of a policy parameter (i.e. $U, s$ or $d$ on the "demand side", or $c$ the cost of harassment on the "supply side") generates multiplier effects in the process of cultural integration and tolerance in society. As a result, having perspective only limited to the side directly affected by the policy generates evaluation bias. Clearly, taking the other side as exogenous will lead to an underestimate effect of the full impact of the policy.

More generally, our results suggest that, while the different integration policies implemented in the US and in Europe ${ }^{26}$ seem to have small effects, this might not be uniquely due to the persistence of segregated neighborhood, but possibly also to the perverse effects of integration policies, which might induce more intense ethnic identities and stronger ethnic

\footnotetext{
${ }^{26}$ For instance, the creations of Zones of Educational Priority (ZEP) and the rehabilitation of bleak housing projects in immigrant neighborhoods under the guise of urban policy ('politique de la ville') in France had very limited effects. See, for example, Bénabou et al. (2009) for an evaluation of the ZEP and Brubaker (2001) who compares the different ways of assimilating ethnic minorities in France, Germany, and the US.
} 
socialization efforts on the part of ethnic parents and extreme behavior from some people from the majority group (see the evidence cited above in Section 8.2).

There are also obvious benefits of integration policies that have not been included in our analysis. For instance, better socio-economic integration of ethnic communities generate economic gains from trade and production. It may also promotes accumulation of human capital of minority communities, with positive growth effects in the economy. Similarly, cultural integration may help the diffusion of "common preferences" which facilitates social consensus on collective decisions and provision of public goods. A full normative discussion of the integration policies would certainly have to take into account these dimensions but this is beyond the scope of the present paper.

Far from supporting policies that establish segregated neighborhoods, this piece of work presented a simple model of cultural evolution which may be a useful block to discuss some issues related to minorities' identity formation and development of oppositional cultures. In particular, it showed that the effect of mixed neighborhood on identity formation and socialization might be perverse. This is particularly so if mixed neighborhoods are conducive of explicit acts of rejection (such as harassment) on the part of the majority group.

\section{References}

[1] Ainsworth-Darnell, J.W., Downey, D.B., 1998. Assessing the oppositional culture explanation for racial/ethnic differences in school performance. American Sociological Review $63,536-553$.

[2] Alba, R.D., 1990. Ethnic Identity: The Transformation of White America. Yale University Press, New Haven.

[3] Akerlof, G.A., 1980. A theory of social custom of which unemployment may be one consequence. Quarterly Journal of Economics 94, 749-775.

[4] Akerlof, G.A., 1997. Social distance and social decisions. Econometrica 65, 1005-1027.

[5] Akerlof, G.A., Kranton, R.E., 2000. Economics and identity. Quarterly Journal of Economics $115,715-753$.

[6] Akerlof, G.A., Kranton, R.E., 2010. Identity Economics: How Our Identities Shape Our Work, Wages, and Well-Being. Princeton University Press, Princeton. 
[7] Alesina, A., La Ferrara, E., 2005. Ethnic diversity and economic performance. Journal of Economic Literature 43, 762-800.

[8] Austen-Smith, D., Fryer, Jr, R.D., 2005. An economic analysis of 'acting white'. Quarterly Journal of Economics 120, 551-583.

[9] Ballester, C., Calvó-Armengol, A., Zenou, Y., 2006. Who's who in networks. Wanted: the key player. Econometrica 74, 1403-1417.

[10] Battu, H., Mwale, M., Zenou, Y., 2007. Oppositional identities and the labor market. Journal of Population Economics 20, 643-667.

[11] Bénabou, R., 1993. Workings of a city: location, education, and production. Quarterly Journal of Economics 108, 619-652.

[12] Bénabou, R., Kramarz, F., Prost, C., 2009. The French zones d'education prioritaire: much ado about nothing? Economics of Education Review 28, 345-356.

[13] Bénabou, R., Tirole, J., 2006. Incentives and prosocial behavior. American Economic Review 96, 1652-1678.

[14] Bénabou, R., Tirole, J., 2011. Identity, morals and taboos: beliefs as assets. Quarterly Journal of Economics, forthcoming.

[15] Bernal, M.E., Knight, G.P., 1993. Ethnic Identity: Formation and Transmission among Hispanics and Other Minorities. State University of New York Press, Albany.

[16] Bernheim, B.D., 1994. A theory of conformity. Journal of Political Economy 102, 841877.

[17] Bisin, A., Verdier, T., 2000. Beyond the melting pot: cultural transmission, marriage, and the evolution of ethnic and religious traits. Quarterly Journal of Economics 115, 955-988.

[18] Bisin, A., Verdier, T., 2001. The economics of cultural transmission and the dynamics of preferences. Journal of Economic Theory 97, 298-319.

[19] Bisin, A., Topa, G., Verdier, T., 2004. Religious intermarriage and socialization in the United States. Journal of Political Economy 112, 615-664. 
[20] Bisin, A., Patacchini, E., Verdier, T., Zenou, Y., 2010. Bend it like Beckham: ethnic identity and integration. CEPR Discussion Paper No. 8054.

[21] Blalock, H., 1956. Economic discrimination and negro increase. American Sociological Review 21, 548-588.

[22] Blalock, H., 1957. Percent mon-white and discrimination in the South. American Sociological Review 22, 677-682.

[23] Blalock, H., 1967. Towards a Theory of Minority-Group Relations. John Wiley and Sons, New York.

[24] Bobo, L.D., 1999. Prejudice as a group position: microfoundations of a sociological approach to racism and racial relations. Journal of Social Issues 55, 445-472.

[25] Boyd, R., Richerson, P.J., 1985. Culture and the Evolutionary Process. University of Chicago Press, Chicago.

[26] Boykin, A.W., 1986. The triple quandary and the schooling of Afro American children. In: Neisser, U. (Ed.), The School Achievement of Minority Children: New Perspectives. Lawrence Erlbaum, HiUandale, NJ.

[27] Bowyer, B.T., 2009. The contextual determinants of white racial attitudes in England. British Journal of Political Science 39, 559-586

[28] Brubaker, R., 2001. The return of assimilation? Changing perspectives on immigration and its sequels in France, Germany, and the United States. Ethnic and Racial Studies $24,531-548$.

[29] Cavalli-Sforza, L.L., Feldman, M.W., 1981. Cultural Transmission and Evolution. Princeton University Press, Princeton.

[30] Cohen-Zada, D., 2006. Preserving religious identity through education: economic analysis and evidence from the US. Journal of Urban Economics 60, 372-398.

[31] Currarini, S., Jackson, M.O., Pin, P., 2009. An economic model of friendship: homophily, minorities, and segregation. Econometrica 77, 1003-1045.

[32] Davey, M., Stone Fish, L., Askew, J., Robila, M., 2003. Parenting practices and the transmission of ethnic identity. Journal of Marital and Family Therapy 29, 195-208. 
[33] Davis, J.B., 1995. Personal identity and standard economic theory. Journal of Economic Methodology 2, 35-52.

[34] Davis, J.B., 2003. The Theory of the Individual in Economics: Identity and Values. Routledge, London.

[35] Davis, J.B., 2004. Complex economic systems: using collective intentionality analysis to explain individual identity in networks. Revue de Philosophie Economique 9, 115-134.

[36] Delpit, L., 1995. Other People's Children: Cultural Conflict in the Classroom. The Free Press, New York.

[37] De Martí, J., Zenou, Y., 2010. Ethnic identity and social distance in friendship formation. Unpublished manuscript, Stockholm University.

[38] Demo, D.H., Hughes, M., 1990. Socialization and racial identity among black Americans. Social Psychology Quarterly 53, 361-374.

[39] De Souza Briggs, X., Popkin, S.J., Goering, J., 2010. Moving to Opportunity. The Story of an American Experiment to Fight Ghetto Poverty. Oxford University Press, Oxford.

[40] Durlauf, S.N., 1996. A theory of persistent income inequality. Journal of Economic Growth 1, 75-93.

[41] Durlauf, S.N., 2004. Neighborhood effects. In: Henderson, J.V., Thisse, J-F., (Eds.), Handbook of Regional and Urban Economics Vol. 4. Elsevier Science, Amsterdam, pp. 2173-2242.

[42] Dustmann, C., Preston, I.P., 2001. Attitudes to ethnic minorities, ethnic context and location decisions. Economic Journal 111, 353-373.

[43] Dustmann, C., Fabbri, F., Preston, I., 2010. Racial harassment, ethnic concentration and economic conditions. IZA Discussion Paper No. 4885, Bonn, Germany.

[44] Fershtman, C., Weiss, Y., 1998. Social rewards, externalities and stable preferences. Journal of Public Economics 70, 53-73.

[45] Fordham, S., Ogbu, J., 1986. Blacks students' school successes: coping with the burden of 'acting white'. The Urban Review 18, 176-206. 
[46] Fryer, R.G. Jr., Torelli, P., 2010. An empirical analysis of 'acting white'. Journal of Public Economics 94, 380-396.

[47] Glaeser, E.L., Scheinkman, J., 2001. Measuring social interactions. In: Durlauf, S.N., Young, H.P. (Eds.), Social Dynamics. MIT Press, Cambridge, MA, pp. 83-132.

[48] Glazer N., Moynihan, D.P., 1970. Beyond the Melting Pot: The Neogroes, Puerto Ricans, Jews, Italians and Irish of New York City. MIT Press, Cambridge, MA.

[49] Harrison, A.O., Wilson, M.N., Pine, C.J., Chan, S.Q., Buriel, R., 1990. Family ecologies of ethnic minority children. Child Development 53, 87-97.

[50] Hughes, M., Demo, D.H., 1989. Self-perceptions of black Americans: self-esteem and personal efficacy. American Journal Sociology 95, 132-159.

[51] Holzer, H.J., Newmark, D., 2000. Assessing affirmative action. Journal of Economic Literature 38, 483-568.

[52] Holzer, H.J., Newmark, D., 2006. Affirmative action: what do we know? Journal of Policy Analysis and Management 25, 463-490.

[53] Horst, U., Kirman, A., Teschl, M., 2007. Changing identity: the emergence of social groups. Unpublished manuscript, GREQAM, Marseilles, France.

[54] Ihlanfeldt, K.R., Scafidi, B., 2002. Black self-segregation as a cause of housing segregation. Evidence from the multi-city study of urban inequality. Journal of Urban Economics 51, 366-390.

[55] Jackson, J. S., McCullough, W.R., Gurin, G., 1981. Family socialization, environment, and identity development in black Americans. In: McAdoo, H. (Ed.), Black Families. Sage Publications, Newbury Park, CA.

[56] Jacoby, T., 1998. Someone Else's House: America's Unfinished Struggle for Integration. Free Press, New York.

[57] Jacoby, T., 1999. Beyond busing. The Wall Street Journal. July 21st.

[58] Jellal, M., Wolff, F.C., 2002. Cultural evolutionary altruism: theory and evidence. European Journal of Political Economy 18, 241-62. 
[59] Johnson, R.C., 1981. The black family and black community development. Journal of Black Psychology 8, 35-52.

[60] Kandel, E., Lazear, E.P., 1992. Peer pressure and partnerships. Journal of Political Economy 100, 801-817.

[61] Kirman, A., Teschl, M., 2004. On the emergence of economic identity. Revue de Philosophie Economique 9, 59-86.

[62] Kirman, A., Teschl, M., 2006. Searching for identity in the capability space. Journal of Economic Methodology 13, 299-325.

[63] Kling, J.R., Ludwig, J., Katz, L.F., 2005. Neighborhood effects on crime for female and male youth: evidence from a randomized housing voucher experiment. Quarterly Journal of Economics 120, 87-130.

[64] Knight, G.P., Bernal, M.E., Cota, M.K., Garza, C.A., Ocampo, K.A., 1993. Family socialization and Mexican American identity and behavior. In: Bernal, M.E. Knight, G.P. (Eds.), Ethnic Identity: Formation and Transmission among Hispanics and Other Minorities. State University of New York Press, Albany, pp. 105-129.

[65] Koszegi, B., 2006. Ego utility, overconfidence, and task choice. Journal of the European Economic Association 4, 673-707.

[66] Lang, K., 2007. Poverty and Discrimination. Princeton University Press, Princeton.

[67] Loury, G.C., 1995. One by One from the Inside Out. Free Press, New York.

[68] Ludwig, J., Duncan, G.J., Hirschfield, P., 2001. Urban poverty and juvenile crime: evidence from a randomized housing-mobility experiment. Quarterly Journal of Economics $116,655-679$.

[69] Ogbu, J.U., 1997. Beyond language: ebonics, proper English and identity in a black American speech community. Unpublished manuscript, University of California at Berkeley, Department of Anthropology.

[70] Oreopoulos, P., 2003. The long-run consequences of living in a poor neighborhood. Quarterly Journal of Economics 4, 1533-75.

[71] Patacchini, E., Zenou, Y., 2011a. Neighborhood effects and parental involvement in the intergenerational transmission of education. Journal of Regional Science. forthcoming. 
[72] Patacchini, E., Zenou, Y., 2011b. Juvenile delinquency and conformism. Journal of Law, Economics, and Organization. forthcoming.

[73] Peters, M. F., 1985. Racial socialization of young black children. In: McAdoo, H.P., McAdoo, J.L. (Eds.), Black Children: Social, Educational, and Parental Environments. Sage Publications, Newbury Park, CA.

[74] Phinney, J.S., 1990. Ethnic identity in adolescents and adults: review of research. Psychological Bulletin 108, 499-514.

[75] Quigley, J.M., Raphael, S., 2008. Neighborhoods, economic self-sufficiency, and the MTO program. Brookings-Wharton Papers on Urban Affairs. 1-46.

[76] Quillian, L., 1995. Prejudice as a response to perceived group threat: population composition and anti-immigrant and racial prejudice in Europe. American Sociological Review $60,586-611$.

[77] Rivera-Batiz, F., Yun, M-S., Gang, I., 2002. Economic strain, ethnic concentration and attitudes towards foreigners in the European Union. Departmental Working Papers 200214, Rutgers University, Department of Economics.

[78] Selod, H., Zenou, Y. 2006. Does city structure affect the labour market outcomes of black workers? Economic Journal 116, 1057-1087.

[79] Sen, A., 1984. Resources, Values and Development. Basil Blackwell, London.

[80] Sen, A., 1993. Capability and well-being. In: Nussbaum, M., Sen, A. (Eds.), The Quality of Life. Clarendon Press, Oxford.

[81] Sen, A., 1999. Reason Before Identity: The Romanes Lecture. Oxford University Press, Oxford.

[82] Shao, M., 2009. A model of social identity with an application to political economy: nation, class, and redistribution. American Political Science Review 103, 147-174.

[83] Spencer, M.B., 1983. Children's cultural values and parental childrearing strategies. Development Review 3, 351-370.

[84] Tajfel, H., 1981. Human Groups and Social Categories: Studies in Social Psychology. Cambridge University Press, Cambridge. 
[85] Taylor, D.M., Lambert, W.E., 1996. The meaning of multiculturalism in a culturally diverse urban American area. Journal of Social Psychology 136, 727-740.

[86] Thornton, M. C., Chatters, L.M., Taylor, R.J., Allen, W.R., 1990. Sociodemographic and environmental correlates of racial socialization by black parents. Child Development 61, 401-409.

[87] Turner J.C., 1982. Towards a cognitive redefinition of the social group," In: Tajfel, H. (Ed.), Social Identity and Intergroup Relations. Cambridge University Press, Cambridge, pp. 15-44.

[88] Whyte, W.F., 1943. Street Corner Society. University of Chicago Press, Chicago.

[89] Wilson, W.J., 1987. The truly Disadvantaged: The Inner City, the Underclass, and Public Policy. University of Chicago Press, Chicago. 


\section{Appendix: Proofs of all the propositions}

Proof of Proposition 1. The interior steady-state equilibrium is defined by (27), which is reproduced here for the clarity of the exposition:

$$
\Omega\left(q_{m}^{*}\right)=q_{m}^{*}\left(1-d+d q_{b}\right)\left(\tau_{m}^{*}-\tau_{o}^{*}\right)+\left(1-\tau_{o}^{*}\right) d\left(1-q_{b}\right)=0
$$

The optimal effort $\tau_{i}^{*}=\tau_{i}\left(q_{m}^{*}\right)$ are defined by $(24)$ and since $\tau_{m}^{*}(1)=0$, evaluating $(27)$ at $q_{m}^{*}=1$ yields:

$$
\Omega(1)=-\left(1-d+d q_{b}\right) \tau_{o}(1)+\left[1-\tau_{o}(1)\right] d\left(1-q_{b}\right)=0
$$

As a result, $\Omega(1)<0$ if and only if:

$$
d\left(1-q_{b}\right)<\tau_{o}(1)
$$

i) Observe that, by $(21), r_{o}(1)=0, Q_{o}(1)=1$ and the optimal effort $\tau_{o}(1)$ defined by $(24)$ can be written as:

$$
\Theta^{\prime}\left(\tau_{o}(1)\right)=\Phi\left(\alpha_{o}\left(I_{o}\right)\right)-U
$$

As a result, condition (37) can be written as:

$$
\Theta^{\prime}\left(d\left(1-q_{b}\right)\right)<\Phi\left(\alpha_{o}\left(I_{o}\right)\right)-U
$$

ii) Furthermore, by differentiating (27), we obtain:

$$
\Omega^{\prime}\left(q_{m}\right)=\left(1-d+d q_{b}\right)\left[\tau_{m}-\tau_{o}+q_{m}\left(\frac{\partial \tau_{m}}{\partial q_{m}}-\frac{\partial \tau_{o}}{\partial q_{m}}\right)\right]-d\left(1-q_{b}\right) \frac{\partial \tau_{o}}{\partial q_{m}}
$$

Therefore, at any interior steady-state equilibrium $q_{m}^{*}$ such that $\Omega\left(q_{m}^{*}\right)=0$, we have:

$$
\Omega^{\prime}\left(q_{m}^{*}\right)=\left(1-d+d q_{b}\right)\left[\tau_{m}^{*}-\tau_{o}^{*}+q_{m}^{*}\left(\frac{\partial \tau_{m}^{*}}{\partial q_{m}}-\frac{\partial \tau_{o}^{*}}{\partial q_{m}}\right)_{q_{m}=q_{m}^{*}}\right]-d\left(1-q_{b}\right)\left(\frac{\partial \tau_{o}}{\partial q_{m}}\right)_{q_{m}=q_{m}^{*}}
$$

Now, from (27), we get:

$$
\left(1-d+d q_{b}\right)\left(\tau_{m}^{*}-\tau_{o}^{*}\right)=-\left(1-\tau_{o}^{*}\right) \frac{d\left(1-q_{b}\right)}{q_{m}^{*}}
$$

Plugging this value in the last equation, we obtain:

$$
\Omega^{\prime}\left(q_{m}^{*}\right)=-\frac{1}{q_{m}^{*}}\left(1-\tau_{o}^{*}\right) d\left(1-q_{b}\right)+q_{m}^{*}\left(1-d+d q_{b}\right)\left(\frac{\partial \tau_{m}^{*}}{\partial q_{m}}-\frac{\partial \tau_{o}^{*}}{\partial q_{m}}\right)_{q_{m}=q_{m}^{*}}-d\left(1-q_{b}\right)\left(\frac{\partial \tau_{o}}{\partial q_{m}}\right)_{q_{m}=q_{m}^{*}}
$$


Since by (25) and (26),

$$
\left(\frac{\partial \tau_{o}}{\partial q_{m}}\right)_{q_{m}=q_{m}^{*}}<0 \text { and }\left(\frac{\partial \tau_{m}^{*}}{\partial q_{m}}-\frac{\partial \tau_{o}^{*}}{\partial q_{m}}\right)_{q_{m}=q_{m}^{*}}<0
$$

$\Omega^{\prime}\left(q_{m}^{*}\right)<0$ at such $q_{m}^{*}$

iii) Also:

$$
\Omega(0)=\left[1-\tau_{o}(0)\right] d\left(1-q_{b}\right)>0
$$

- Now suppose that:

$$
\Theta^{\prime}\left(d\left(1-q_{b}\right)\right)<\Phi\left(\alpha_{o}\left(I_{o}\right)\right)-U
$$

which implies that $\Omega(1)<0$ (see (38)). Then because of iii) and the continuity of $\Omega\left(q_{m}\right)$, there exists at least one $\left.q_{m}^{*} \in\right] 0,1\left[\right.$ such that $\Omega\left(q_{m}^{*}\right)=0$. At such point because of ii) we have $\Omega^{\prime}\left(q_{m}^{*}\right)<0$ which implies that $q_{m}^{*}$ is unique.

- Now, suppose on the opposite, that

$$
\Theta^{\prime}\left(d\left(1-q_{b}\right)\right)>\Phi\left(\alpha_{o}\left(I_{o}\right)\right)-U
$$

then $\Omega(1)>0$. Suppose that there exists an interior solution $\left.q_{m}^{*} \in\right] 0,1[$ such that $\Omega\left(q_{m}^{*}\right)=0$. Then, by the same token ii), it should be unique and the continuity of $\Omega($.$) implies that, for all points q_{m}>q_{m}^{*}$, one has $\Omega\left(q_{m}\right)<0$, contradicting the fact that $\Omega(1)>0$.

It follows that a necessary and sufficient condition to have an interior (unique) steady state $q_{m}^{*}$ is condition (38)

Finally, it is immediate to see that, for an interior solution $q_{m}^{*}$, we have:

$$
\tau_{m}^{*}-\tau_{o}^{*}=-\frac{\left(1-\tau_{o}^{*}\right) d\left(1-q_{b}\right)}{q_{m}^{*}\left(1-d+d q_{b}\right)} \leq 0
$$

which means that oppositional families exert more socialisation effort than mainstream families.

Proof of Proposition 2. Using (22), (23), (25) and (26), let us write the function $\Omega($.$) ,$ defined in (27), as depending on all the parameters, i.e.

$$
\begin{aligned}
\Omega\left(q_{m}, q_{b}, s, d, U\right)= & q_{m}\left(1-d+d q_{b}\right)\left[\tau_{m}\left(\bar{q}_{m}, \stackrel{+}{q_{b}}, \bar{s}, \bar{d}, \stackrel{+}{U}\right)-\tau_{o}\left(\stackrel{+}{q}_{m}, \overline{q_{b}},+\stackrel{+}{s}, \stackrel{\vec{d}}{U}\right)\right] \\
& +\left[1-\tau_{o}\left(\stackrel{+}{q}_{m}, \bar{q}_{b}, \stackrel{+}{s}, \stackrel{+}{d}, \bar{U}\right)\right] d\left(1-q_{b}\right)
\end{aligned}
$$


From this equation, it is straightforward to see that for $v \in\left\{U, s, q_{b}, d\right\}$, we have:

$$
\frac{\partial q_{m}^{*}}{\partial v}=-\frac{\partial \Omega / \partial v}{\partial \Omega / \partial q_{m}}
$$

which has the sign of $\partial \Omega / \partial v$ since $\partial \Omega / \partial q_{m}<0$. The first two results follow from:

$$
\begin{aligned}
& \frac{\partial \Omega}{\partial U}=q_{m}\left(1-d+d q_{b}\right)\left(\frac{\partial \tau_{m}}{\partial U}-\frac{\partial \tau_{o}}{\partial U}\right)-\frac{\partial \tau_{o}}{\partial U} d\left(1-q_{b}\right)>0 \\
& \frac{\partial \Omega}{\partial s}=q_{m}\left(1-d+d q_{b}\right)\left(\frac{\partial \tau_{m}}{\partial s}-\frac{\partial \tau_{o}}{\partial s}\right)-\frac{\partial \tau_{o}}{\partial s} d\left(1-q_{b}\right)<0
\end{aligned}
$$

while the ambiguity results come from:

$$
\frac{\partial \Omega}{\partial q_{b}}=q_{m} d\left(\tau_{m}^{*}-\tau_{o}^{*}\right)-\left(1-\tau_{o}^{*}\right) d+q_{m}\left(1-d+d q_{b}\right)\left(\frac{\partial \tau_{m}}{\partial q_{b}}-\frac{\partial \tau_{o}}{\partial q_{b}}\right) \gtreqless 0
$$

and

$$
\frac{\partial \Omega}{\partial d}=-q_{m}\left(1-q_{b}\right)\left(\tau_{m}^{*}-\tau_{o}^{*}\right)+\left(1-\tau_{o}^{*}\right)\left(1-q_{b}\right)+q_{m}\left(1-d+d q_{b}\right)\left(\frac{\partial \tau_{m}}{\partial q_{b}}-\frac{\partial \tau_{o}}{\partial q_{b}}\right) \gtreqless 0
$$

since, in equilibrium, $\tau_{m}^{*}-\tau_{o}^{*} \leq 0$.

Proof of Corollary 2. Assume that the socialization costs are convex enough, i.e. bounded from below by some positive constant $K: \Theta^{\prime \prime}\left(\tau_{i}\right)>K$, and consider then a marginal increase in $d$. When $d=0$, the long run cultural equilibrium is given by the condition:

$$
\tau_{m}^{*}-\tau_{o}^{*}=0
$$

which translates into (see equation (30)):

$$
\frac{q_{m}^{*}}{1-q_{m}^{*}}=\frac{\Delta V_{m}\left(\bar{q}_{m}^{-}, \bar{s}, \stackrel{+}{q_{b}}\right)}{\Delta V_{o}\left(\left(_{m}^{+}, \stackrel{+}{s}, \overline{q_{b}}\right)\right.}
$$

For $d=0$, we have:

$$
\frac{\partial q_{m}^{*}}{\partial d}=-\frac{1}{\partial \Omega / \partial q_{m}}\left\{\left(1-\tau_{o}^{*}\right)\left(1-q_{b}\right)+q_{m}^{*}\left(\frac{\partial \tau_{m}^{*}}{\partial d}-\frac{\partial \tau_{o}^{*}}{\partial d}\right)\right\}
$$

with

$$
\frac{\partial \tau_{m}^{*}}{\partial d}=\frac{-\left(1-q_{m}^{*}\right)\left(1-q_{b}\right) \Delta V_{m}}{\Theta^{\prime \prime}\left(\tau_{m}^{*}\right)}
$$

and

$$
\frac{\partial \tau_{o}^{*}}{\partial d}=\frac{\left(1-q_{m}^{*}\right)\left(1-q_{b}\right) \Delta V_{o}}{\Theta^{\prime \prime}\left(\tau_{o}^{*}\right)}
$$


Therefore,

$$
\begin{aligned}
\frac{\partial q_{m}^{*}}{\partial d} & =-\frac{\left(1-q_{b}\right)}{\partial \Omega / \partial q_{m}}\left\{\left(1-\tau_{o}\right)-q_{m}^{*}\left(1-q_{m}^{*}\right)\left[\frac{\Delta V_{m}}{\Theta^{\prime \prime}\left(\tau_{m}^{*}\right)}+\frac{\Delta V_{o}}{\Theta^{\prime \prime}\left(\tau_{o}^{*}\right)}\right]\right\} \\
& =-\frac{\left(1-q_{b}\right)}{\partial \Omega / \partial q_{m}}\left\{\left(1-\tau_{o}\right)-q_{m}^{*}\left(1-q_{m}^{*}\right) \frac{\Delta V_{m}+\Delta V_{o}}{\Theta^{\prime \prime}\left(\tau^{*}\right)}\right\}
\end{aligned}
$$

as $\tau_{m}^{*}=\tau_{o}^{*}=\tau^{*}$ and $\Theta^{\prime \prime}\left(\tau_{m}^{*}\right)=\Theta^{\prime \prime}\left(\tau_{o}^{*}\right)=\Theta^{\prime \prime}\left(\tau^{*}\right)$. Therefore

$$
\frac{\partial q_{m}^{*}}{\partial d}>-\frac{\left(1-q_{b}\right)}{\partial \Omega / \partial q_{m}}\left\{\left(1-\tau_{o}\right)-q_{m}^{*}\left(1-q_{m}^{*}\right) \frac{\Delta V_{m}+\Delta V_{o}}{K}\right\}
$$

Consider now the sign of:

$$
1-\tau_{o}-\frac{q_{m}\left(1-q_{m}\right)\left(\Delta V_{m}+\Delta V_{o}\right)}{K}
$$

Recalling that $\Theta^{\prime}\left(\tau_{o}\right)=q_{m} \Delta V_{m}$ and given (39), we obtain:

$$
\begin{aligned}
1-\tau_{o}-\frac{q_{m}\left(1-q_{m}\right)\left(\Delta V_{m}+\Delta V_{o}\right)}{K} & =1-\Theta^{\prime-1}\left(\frac{\Delta V_{m} \Delta V_{o}}{\Delta V_{m}+\Delta V_{o}}\right)-\frac{1}{\Theta^{\prime \prime}\left(\tau_{m}\right)} \frac{\Delta V_{m} \Delta V_{o}}{\Delta V_{m}+\Delta V_{o}} \\
& >1-\Theta^{\prime-1}\left(\frac{\Delta V_{m} \Delta V_{o}}{\Delta V_{m}+\Delta V_{o}}\right)-\frac{1}{K} \frac{\Delta V_{m} \Delta V_{o}}{\Delta V_{m}+\Delta V_{o}}
\end{aligned}
$$

Now, define the following function:

$$
\varphi(x)=1-\Theta^{\prime-1}(x)-\frac{1}{K} x
$$

This function is such that for all $x, \varphi^{\prime}(x)<0$, and $\varphi(x)>1-\frac{2}{K} x$. Thus for $K>2$, we get $\varphi(1)>0$ and $\varphi(x)>0$. It follows that, for $K>2$, we have that

$$
\frac{\Delta V_{m} \Delta V_{o}}{\Delta V_{m}+\Delta V_{o}}<1
$$

is a sufficient condition for

$$
1-\tau_{o}-\frac{q_{m}\left(1-q_{m}\right)\left[\left(\Delta V_{m}+\Delta V_{o}\right)\right.}{K}>0
$$

Therefore, from (40) a sufficient condition for $\frac{\partial q_{m}^{*}}{\partial d}>0$ for $d \approx 0$ is also:

$$
\frac{\Delta V_{m} \Delta V_{o}}{\Delta V_{m}+\Delta V_{o}}<1
$$

But it then easy to see that:

$$
\frac{\Delta V_{m} \Delta V_{o}}{\Delta V_{m}+\Delta V_{o}}=\frac{\left[U+\Psi\left(\alpha_{m}\left(q_{m}\right)\right)\right]\left[\Phi\left(\alpha_{o}\left(q_{m}\right)-U\right]\right.}{\Phi\left(\left(\alpha_{o}\left(q_{m}\right)\right)+\Psi\left(\alpha_{m}\left(q_{m}\right)\right)\right)}<\frac{\left[U+\Psi\left(\alpha_{m}(0)\right)\right]\left[\Phi\left(\alpha_{o}(1)-U\right]\right.}{\Phi\left(\left(\alpha_{o}(0)\right)\right.}
$$


using the fact that $\alpha_{m}\left(q_{m}\right)$ and $\alpha_{o}\left(q_{m}\right)$ are respectively decreasing and increasing in $q_{m}$ and that $\alpha_{m}(1)=0$. Thus a sufficient condition for

$$
\frac{\Delta V_{m} \Delta V_{o}}{\Delta V_{m}+\Delta V_{o}}<1
$$

can be written as

$$
\frac{\left[U+\Psi\left(\alpha_{m}(0)\right)\right]\left[\Phi\left(\alpha_{o}(1)\right)-U\right]}{\Phi\left(\left(\alpha_{o}(0)\right)\right.}<1
$$

which is satisfied for all values of $U>0$ when

$$
\frac{\left[\Phi\left(\alpha_{o}(1)\right)+\Psi\left(\alpha_{m}(0)\right)\right]^{2}}{4 \Phi\left(\left(\alpha_{o}(0)\right)\right.}<1
$$

Finally, it follows that $\frac{\partial q_{m}^{*}}{\partial d}>0$ for $d \approx 0$ when parameters values satisfy (42) and $K>2$.

Proof of Corollary 3. The proof is immediate and comes simply from the fact that an increase in $U$ has no direct impact on the probabilities $Q_{i}$ but negatively affects the prevalence of oppositional types in the long run (ie. $\frac{d q_{m}^{*}}{d U}>0$ ).

Proof of Corollary 4: We know that in the case of $d=0$, we have:

$$
q_{m}^{*}=\frac{U+\Psi\left(\alpha_{m}\right)}{\Psi\left(\alpha_{m}\right)+\Phi\left(\alpha_{o}\right)}
$$

Simple differentiation for a parameter $v \in\left\{U, s, q_{b}, d\right\}$ yields:

with

$$
\frac{d q_{m}^{*}}{d v}=\frac{\frac{\partial U}{\partial v} \frac{1}{\Psi+\Phi}+A \frac{\partial Q_{m}}{\partial v}}{1+A\left(1-s+s q_{b}\right)}
$$

and thus

$$
A=\frac{\Psi^{\prime}\left(\alpha_{m}\right) \Phi\left(\alpha_{o}\right) \frac{d \alpha_{m}}{d Q_{m}}}{\left[\Psi\left(\alpha_{m}\right)+\Phi\left(\alpha_{o}\right)\right]^{2}}+\frac{\left.\Phi^{\prime}\left(\alpha_{o}\right)\left[U+\Psi\left(\alpha_{m}\right)\right)\right] \frac{d \alpha_{o}}{d Q_{o}}}{\left.\left[\Psi\left(\alpha_{m}\right)\right)+\Phi\left(\alpha_{o}\right)\right]^{2}}>0
$$

$$
\begin{aligned}
\frac{d \alpha_{m}}{d v} & =\frac{d \alpha_{m}}{d Q_{m}}\left[\frac{\partial Q_{m}}{\partial v}-\left(1-s+s q_{b}\right) \frac{\frac{\partial U}{\partial v} \frac{1}{\Psi+\Phi}+A \frac{\partial Q_{m}}{\partial v}}{1+A\left(1-s+s q_{b}\right)}\right] \\
& =\frac{d \alpha_{m}}{d Q_{m}}\left[\frac{\frac{\partial Q_{m}}{\partial v}-\left(1-s+s q_{b}\right) \frac{\partial U}{\partial v} \frac{1}{\Psi+\Phi}}{1+A\left(1-s+s q_{b}\right)}\right]
\end{aligned}
$$


Similarly:

$$
\begin{aligned}
\frac{d \alpha_{o}}{d v} & =\frac{d \alpha_{o}}{d Q_{o}}\left[\frac{\partial Q_{o}}{\partial v}+\left(1-s+s q_{b}\right) \frac{\frac{\partial U}{\partial v} \frac{1}{\Psi+\Phi}-A \frac{\partial Q_{o}}{\partial v}}{1+A\left(1-s+s q_{b}\right)}\right] \\
& =\frac{d \alpha_{o}}{d Q_{o}}\left[\frac{\frac{\partial Q_{o}}{\partial v}+\left(1-s+s q_{b}\right) \frac{\partial U}{\partial v} \frac{1}{\Psi+\Phi}}{1+A\left(1-s+s q_{b}\right)}\right]
\end{aligned}
$$

Given that

$$
\begin{gathered}
Q_{m}=Q_{m}\left(\overline{q_{m}}, \bar{s}, \stackrel{+}{q_{b}}\right)=\left(1-s+s q_{b}\right)\left(1-q_{m}\right) \\
Q_{o}=Q_{o}\left(\stackrel{+}{q_{m}}, \stackrel{+}{s}, \overline{q_{b}}\right)=\left(1-s+s q_{b}\right) q_{m}+s\left(1-q_{m}\right)
\end{gathered}
$$

and $\frac{\partial U}{\partial s}=0$, we immediately obtain:

$$
\begin{aligned}
\frac{d \alpha_{m}}{d s} & =\frac{d \alpha_{m}}{d Q_{m}}\left[\frac{\frac{\partial Q_{m}}{\partial s}}{1+A\left(1-s+s q_{b}\right)}\right] \\
\frac{d \alpha_{o}}{d s} & =\frac{d \alpha_{o}}{d Q_{o}}\left[\frac{\frac{\partial Q_{o}}{\partial s}}{1+A\left(1-s+s q_{b}\right)}\right]
\end{aligned}
$$

which correspond to the relations stated in the Corollary.

Formally,

$$
\begin{gathered}
\frac{d \alpha_{m}^{*}}{d Q_{m}} \frac{\partial Q_{m}}{\partial s}<\frac{d \alpha_{m}^{*}}{d s}<0 \\
0<\frac{d \alpha_{o}^{*}}{d s}<\frac{d \alpha_{o}^{*}}{d Q_{o}} \frac{\partial Q_{o}}{\partial s}
\end{gathered}
$$


Figure 1: Full cultural segmentation equilibrium $(d=0)$

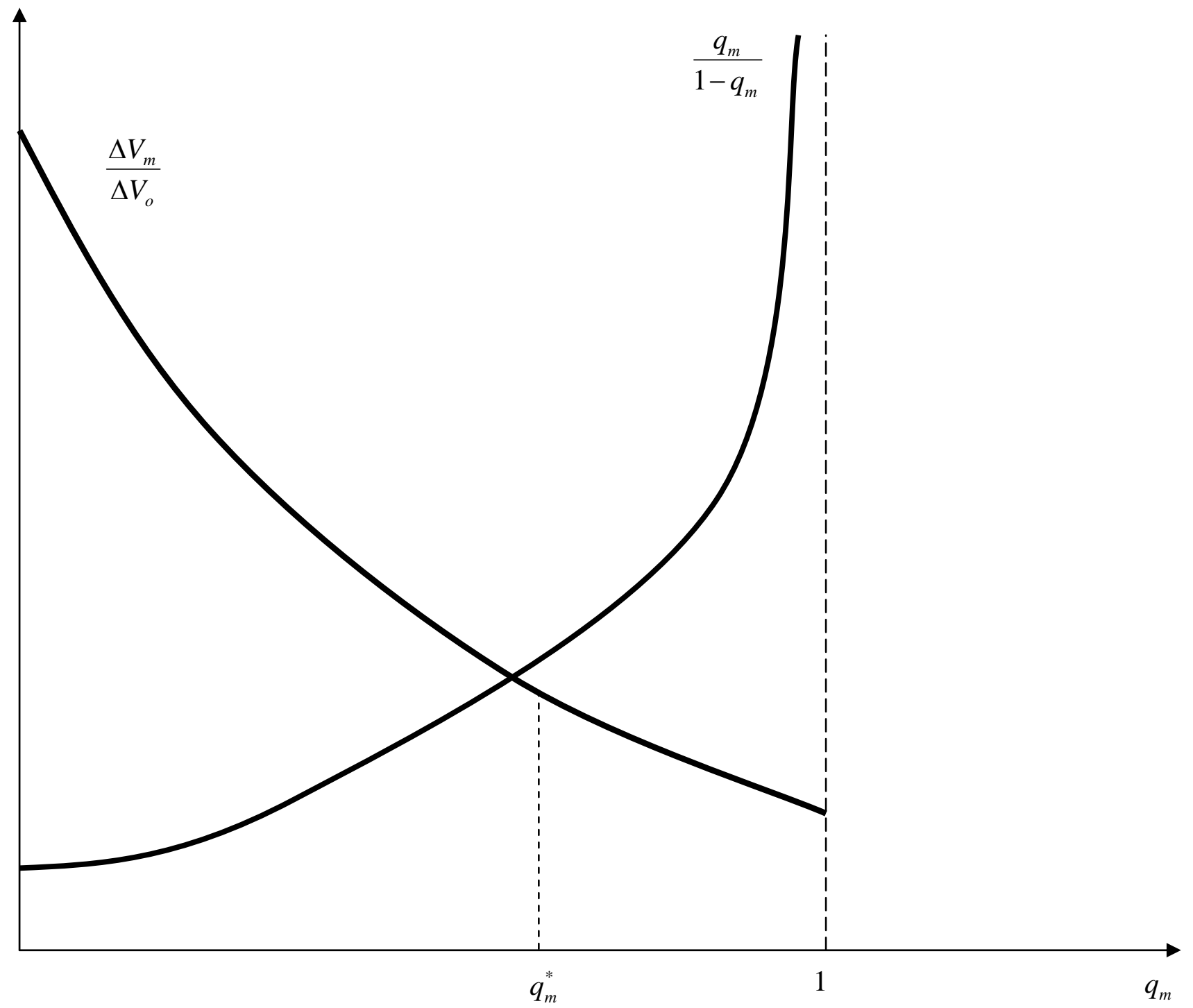


Figure 2a: Identity and conditional harassment

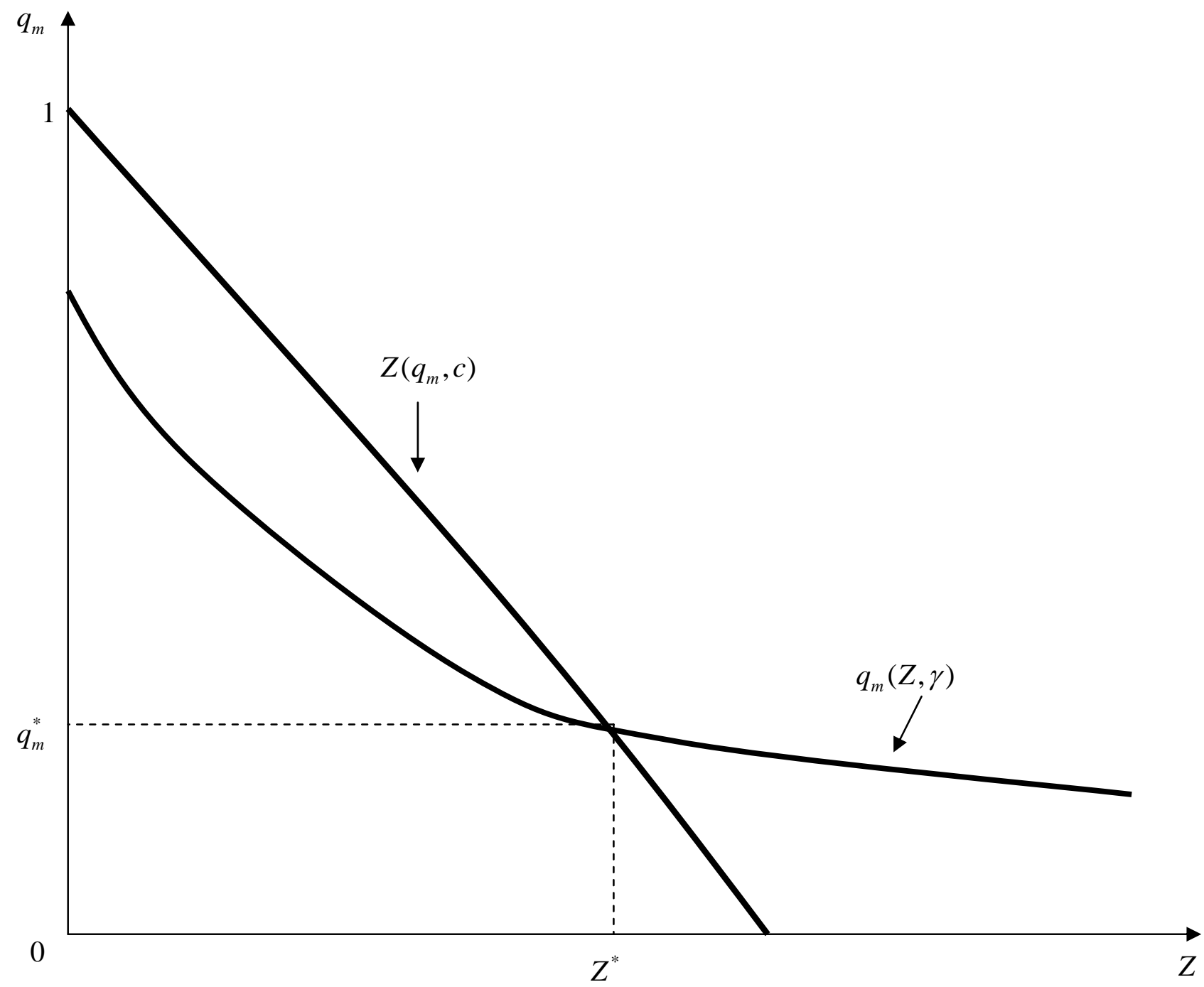




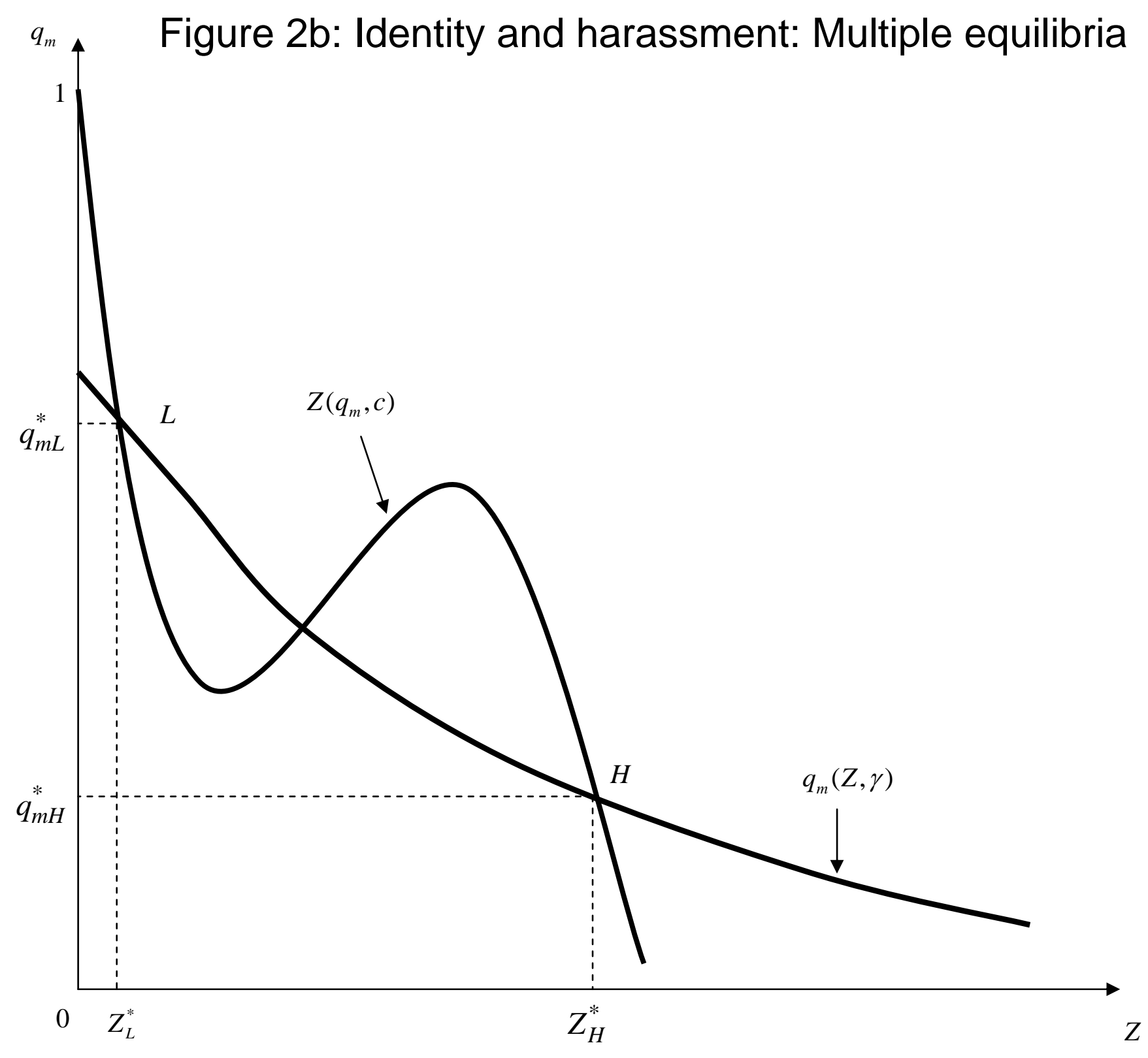


Figure 3a: "Conditional" racism and oppositional cultures

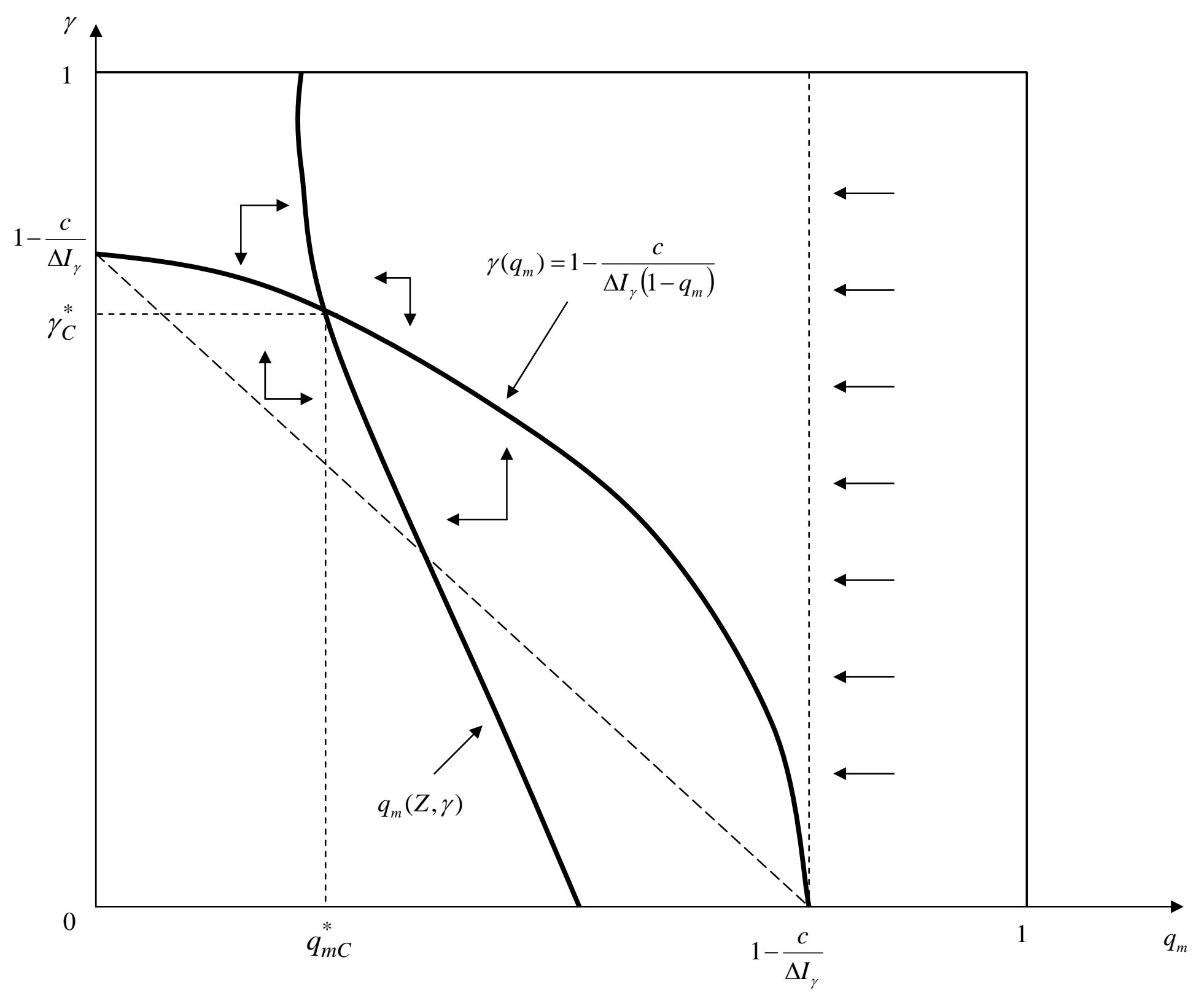


Figure 3b: "Conditional" racism and oppositional cultures: Multiple equilibria

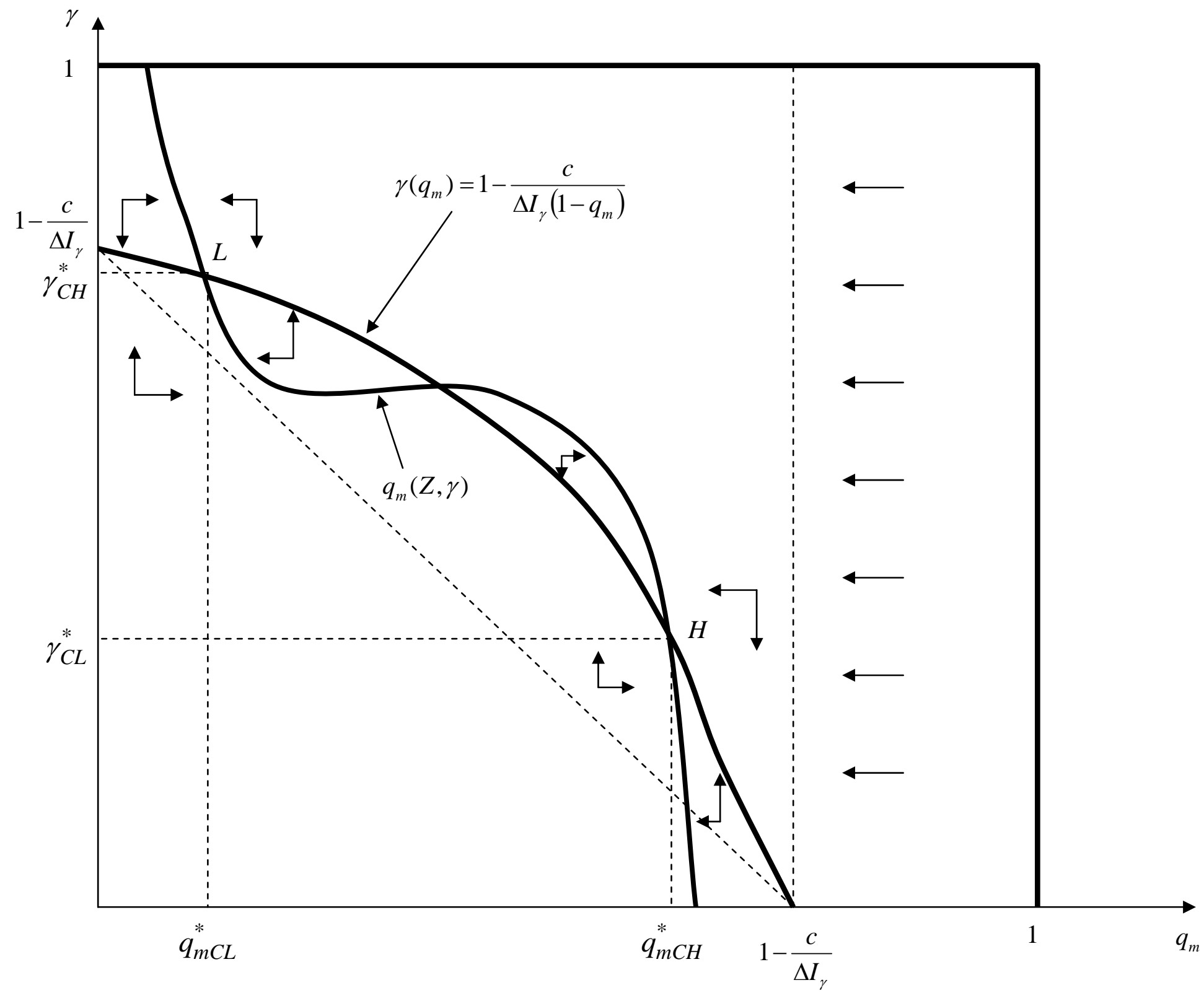

